NBER WORKING PAPER SERIES

\title{
DECLINING WORKER TURNOVER: \\ THE ROLE OF SHORT DURATION EMPLOYMENT SPELLS
}

\author{
Michael J. Pries \\ Richard Rogerson \\ Working Paper 26019 \\ http://www.nber.org/papers/w26019 \\ NATIONAL BUREAU OF ECONOMIC RESEARCH \\ 1050 Massachusetts Avenue \\ Cambridge, MA 02138 \\ June 2019
}

We are thankful for helpful comments and suggestions from seminar participants at the Federal Reserve Bank of Cleveland and the 2019 MidWest Macro Conference at the University of Georgia. The views expressed herein are those of the authors and do not necessarily reflect the views of the National Bureau of Economic Research.

At least one co-author has disclosed a financial relationship of potential relevance for this research. Further information is available online at http://www.nber.org/papers/w26019.ack

NBER working papers are circulated for discussion and comment purposes. They have not been peer-reviewed or been subject to the review by the NBER Board of Directors that accompanies official NBER publications.

(C) 2019 by Michael J. Pries and Richard Rogerson. All rights reserved. Short sections of text, not to exceed two paragraphs, may be quoted without explicit permission provided that full credit, including ( $)$ notice, is given to the source. 
Declining Worker Turnover: the Role of Short Duration Employment Spells

Michael J. Pries and Richard Rogerson

NBER Working Paper No. 26019

June 2019

JEL No. E24,J23

\section{ABSTRACT}

Using the Quarterly Workforce Indicators, we document that a significant amount of the decline in labor market turnover during the last two decades is accounted for by the decline in employment spells that last less than a quarter. Using a search and matching model that incorporates noisy signals about the quality of a worker-firm match, we show that improved candidate screening by firms can account for the decline in short-lived employment spells. Quantitative exercises show that this explanation can account for the observed changes in various labor market outcomes, whereas alternative potential explanations, such as increased hiring costs, cannot.

Michael J. Pries

Department of Economics

3034 Jenkins and Nanovic Halls

University of Notre Dame

Notre Dame, IN 46556

mpries@nd.edu

Richard Rogerson

Department of Economics

JRR Building Room 292

Princeton University

Princeton, NJ 08544

and NBER

rdr@princeton.edu 


\section{Introduction}

Following the recent literature documenting a large secular decline in labor market flows in the US over the past few decades (see, for example, Davis and Haltiwanger (2014), Hyatt and Spletzer (2013), and Molloy et al. (2016)), researchers have sought to understand the source of this decline. ${ }^{1}$ One strand of this literature assesses the extent to which this declining "dynamism" represents a reduction in the responsiveness of incumbent firms to changing circumstances or of entrepreneurs to new possibilities. (See, for example, the recent paper by Engbom (2018)). In this paper, we present evidence that an additional and novel channel also plays a quantitatively significant role. In particular, we argue that changes in the manner in which firms hire new employees have also made an important contribution to the decline in labor market flows. These changes result in newly formed matches that are of higher expected quality and therefore more likely to endure. This increase in the quality of newly formed matches leads to less subsequent turnover and can explain an important part of the overall decline in labor market flows.

The starting point for our analysis is the observation that much of the decline in worker turnover in the US labor market is accounted for by a decrease in employment spells of very short (less than a quarter) duration. Using data from the QWI (Quarterly Workforce Indicators), we document that in 1999, spells that lasted a quarter or less accounted for about $8 \%$ of all employment spells, and that by 2015 , this figure had fallen to $4.9 \% .^{2}$ This decline in employment spells that end in their first quarter accounted for more than half $(55 \%)$ of the overall $27.6 \%$ decline in the rate of separations.

This reduction in short-duration employment spells is pervasive; that is, it is not due to shifting demographics or to changes in industry or firm-size composition. As such, it must be the result of changes in the labor market environment - changes in search frictions, changes in policy, or some other change in how the labor market functions. To assess these possibilities we develop a version of the Diamond-Mortensen-Pissarides (DMP) model that can account for the salient features of the employment spell distribution in the US.

Our model differs from simple DMP models along two dimensions. First, when a worker and firm meet they observe a noisy signal about match quality. Second, matches are "experience goods" in the sense that the quality of the match is revealed over time as production takes place. This second feature gives rise to a match hazard rate that declines rapidly with match tenure, and allows us to capture the key features of the empirical relationship between tenure and hazard rates. In the model's equilibrium, a firm and worker will choose

\footnotetext{
${ }^{1}$ This literature has also documented a secular decline in several measures of establishment and firm dynamics as well. See, for example, Decker et al. (2017), and Pugsley and Sahin (2018).

${ }^{2}$ Both of these numbers are higher - slightly over $10 \%$ in 1999 and about $5.5 \%$ in 2015 -if one uses a point in time measure of employment, rather than total number of employment spells in a quarter, in the denominator of the rate calculation, as discussed in section 2 .
} 
to form a match upon meeting only if the value of the noisy signal exceeds some threshold. Changes in the equilibrium value of this threshold influence the average quality of newly formed matches, and thus influence the hazard rates of newly formed matches.

Our model yields a simple three parameter stochastic process that summarizes the dynamics of employment spells in steady state. One of the parameters reflects the overall turnover rate in the labor market, i.e., a constant rate at which matches exogenously break up. This captures both the job creation/destruction process and the rate at which workers separate to pursue other options. The other two parameters characterize the quality of newly formed matches and the speed with which true match quality is revealed. We show that this simple three parameter process can capture the salient empirical properties of the employment spell distribution in the late 1990s. We then use this representation to infer what changes in these three parameters are required to account for the observed changes in worker turnover that occurred in the subsequent two decades; in particular, the large changes in the hazard rates of low-tenure employment spells. While we find the largest source of the observed drop in turnover is a decrease in the parameter reflecting the overall level of turnover in the economy, we find that it accounts for only $60 \%$ of the observed drop, with most of the remaining $40 \%$ accounted for by the parameter that captures the average quality of newly formed matches.

We then use a calibrated version of our model to examine what changes in primitives can empirically account for the change in observed match quality of newly formed matches in a reasonable way. Our model suggests three intuitive mechanisms that can lead to this. First, if the search process that brings workers and firms together becomes more efficient, then workers and firms will be more discriminating when deciding which matches to form. Second, an increase in training or start-up costs incurred after a match is formed will also lead workers and firms to become more selective, as they will want to avoid paying these costs for matches that later turn out to be unprofitable. Lastly, if the initial signal about match quality is of higher quality (less noisy), then firms and workers will be more able to weed out unprofitable matches ex ante.

While improvements in search efficiency or increases in training or start-up costs associated with hiring can both lead to a higher threshold for match formation, and hence a higher quality of newly formed matches, we find that each of these explanations would also imply changes in the job-finding rate and unemployment rate that are so large as to render these explanations empirically implausible. ${ }^{3}$ In contrast, we show that a decrease in the variance of the noisy signal, i.e. an increase in the accuracy of the initial signal, can

\footnotetext{
${ }^{3}$ Two other possible sources of an increase in the threshold are an increase in worker dismissal costs or a decrease in firm recruiting costs. An increase in dismissal costs has implications that are similar to those of an increase in start-up costs, and a decrease in recruiting costs is very similar to an increase in match efficiency.
} 
account for the improvement in average quality of newly formed matches with empirically reasonable effects on both the worker job-finding rate and the unemployment rate.

Our finding of an important role for a more efficient screening process for new hires is consistent with several studies that looked at changes in the ways that firms screen and hire workers. Advances in information technology and data analytics have allowed employers to rely more heavily on "workforce analytics" and worker screening tests to identify workers better suited for particular jobs, or to identify potential worker shortcomings before making a hire. For example, Autor and Scarborough (2008) explore the increased utilization of testbased worker screening by examining data from a large national retail chain that adopted an electronic application that incorporated a computer-administered personality test. Of note, the short-lived jobs emphasized in the present paper are common at this retail chain, with median and mean job durations of 99 and 174 days, respectively. Autor and Scarborough (2008) find that the introduction of the worker screens increased the mean and median durations by between 10 and 12\%. Hoffman, Kahn and Li (2017) similarly examine worker turnover among a sample of service sector firms that rely on worker analytics screening tests that make hiring recommendations based on "a large battery of questions, including those on computer/technical skills, personality, cognitive skills, fit for the job, and various job scenarios." They find that the utilization of these methods increases job tenures by just over $25 \%$.

Our analysis suggests a nuanced perspective on the decline in worker turnover may be required. Whereas the literature has tended to ask whether this decline reflects negative or benign influences, our analysis suggests that the overall effect may well reflect a combination of effects that may differ in their effect on welfare. Improvements in information technology may have led to more efficient hiring decisions as modelled here, at the same time that other factors have decreased the diffusion of technological innovation, as suggested by Akcigit and Ates (2019).

The model studied in this paper is closely related to the model we developed in Pries and Rogerson (2005). The key difference is that here we explicitly model the information received at the time of a meeting and how this affects the distribution of priors regarding match quality. Our analysis is also related to complementary work in Mercan (2017). While focusing on a different data sources, and more specifically on the observed decline job-tojob transitions, Mercan (2017) also argues that more precise initial information about the quality of job matches is an important factor in accounting for the reduction in labor market flows over the last two decades.

The rest of the paper is organized as follows. The next section documents the key facts about the decline in worker turnover and the role of short duration employment spells in accounting for this change. Section 3 considers several possible explanations for the decline 
in short duration employment spells. Section 4 presents our general equilibrium model of match formation and derives the three parameter process that fully characterizes the distribution of employment spells in steady state. Section 5 uses this reduced form process to shed light on what changes in those parameters are needed to account for the decline in worker turnover patterns in the US between 1999 and 2015. Section 6 then uses a calibrated version of our model to assess what primitives are best able to generate the needed increase in the quality of newly formed matches. Section 7 concludes.

\section{Evidence on Changes in Labor Market Turnover}

In this section we use data from the Quarterly Workforce Indicators (QWI) to document several changes in the nature of labor market turnover during the last two decades.

\subsection{Data}

The QWI is a publicly available database that provides information on employment flows based on microdata in the Longitudinal Employer-Household Dynamics (LEHD) database. The LEHD in turn uses quarterly state unemployment insurance (UI) records to identify worker-firm employment spells.

The data utilized here covers 30 states for a period of 17 years, from the first quarter of 1999 to the second quarter of 2015 . These 30 states account for about $65 \%$ of national employment; incorporating data prior to 1999Q1 would require that our data coverage be limited to a smaller number of states. The QWI provides state-level information on various measures of employment, hires, and separations that can be disaggregated by worker characteristics (age, sex, educational attainment, race, and ethnicity) and firm characteristics (industry, firm age, and firm size). Greater detail on the data and on the construction of the various measures of labor market turnover that are used in this section are given in the data appendix.

The QWI allows us to calculate two important measures of turnover. First, we can measure the total number of new spells that begin within a given quarter. These are worker-firm pairs that show earnings to the state UI agency in one quarter, but show no such earnings in the preceding quarter. We will refer to this as the number of hires in period $t$ and denote it by $H_{t}$. Similarly, separations in period $t$, denoted as $S_{t}$, are identified as worker-firm pairs that show earnings reported in quarter $t$, but not in quarter $t+1$. If we let $N_{t}$ denote the total number of spells in quarter $t$, then we can calculate a separations rate $s_{t}=S_{t} / N_{t}$ and a hires rate $h_{t}=H_{t} / N_{t}{ }^{4}$

\footnotetext{
${ }^{4} \mathrm{An}$ alternative measure that could be used in the denominator would be the average of the beginningof-quarter employment and the end-of-quarter employment. By construction, this measure will be less than
} 
Importantly for our purposes, the QWI also allows us to measure the incidence of spells of different duration, as well as the hazard rates at which spells of different duration come to an end. In particular, we can identify employment spells that belong to three different (current) tenure categories: one quarter, two quarters, or three-plus quarters. While it would be ideal if we could identify employment spells of all tenure levels, rather than having to lump together all spells with three or more quarters of tenure, the information that we do have is still sufficient to allow us to investigate the importance of the changing incidence of employment spells of short duration, which is our primary focus.

The counts for the number of worker-firm pairs in quarter $t$ in the three tenure categories are denoted by $N_{i t}$, for $i=1,2,3$. Note that $N_{1 t}=H_{t}$. The number of employment spells in the second tenure category, $N_{2 t}$, are worker-firm pairs with earnings reported for quarters $t$ and $t-1$, but not for $t-2$. The number of spells in the last tenure category, $N_{3 t}$, (in this instance the 3 in the notation denotes three-plus quarters of tenure) consists of all other worker-firm pairs.

Whereas the $N_{i t}$ counts tell us the number of employments spells with a particular current tenure level, we can also identify the number of spells in period $t$ whose final tenure is either one, two, or three-plus quarters. That is, among the spells that are in their first quarter of tenure, $N_{1 t}$, we can identify the spells that also end during that first quarter as those spells in $N_{1 t}$ that do not report any earnings in period $t+1$. We let $Q_{1 t}$ denote the number of these spells that last just one quarter. In a similar manner, among the $N_{2 t}$ spells that are in their second quarter, we identify those that end in the quarter as the worker-firm pairs that do not report earnings in period $t+1$, and we let $Q_{2 t}$ denote the number of these spells. Finally, we let $Q_{3 t}$ denote employment spells with three or more quarters of tenure that come to an end in period $t$.

For the one quarter and two quarter categories, we can use these counts to calculate two different rates of interest. First, we can calculate incidence rates. That is, we let $q_{1 t}$ denote the fraction of total spells in period $t$ that are one quarter spells: $q_{1 t}=Q_{1 t} / N_{t}$. Similarly, $q_{2 t}=Q_{2 t} / N_{t}$ is the incidence rate of spells that last just two quarters (with period $t$ being their last quarter). These incidence rates tell us what share of overall employment is accounted for by short duration spells.

Second, we can calculate hazard rates for the tenure categories (i.e. the rates at which spells of different tenure levels come to an end). We let $\delta_{1 t}$ denote this hazard rate for one quarter spells, and it is calculated as the fraction of spells currently in their first quarter that also end in that first quarter: $Q_{1 t} / N_{1 t}$. Similarly, $\delta_{2 t}=Q_{2 t} / N_{2 t}$ and $\delta_{3 t}=Q_{3 t} / N_{3 t}$ denote the hazard rate for two quarter and three-plus quarter spells.

the total number of employment spells, since it excludes spells that begin and end in the same quarter. While the difference between the two measures affects the level of the various calculated rates, it does not affect the trend, which is the primary focus of this paper. 
Note that the three tenure categories that we utilize refer to how many boundaries (between measurement quarters) a spell crosses and not the actual duration of the spell. According to our definitions, a spell that begins in the last week of one quarter and ends in the first week of the subsequent quarter will count as a two quarter spell even though its duration is less than one quarter. It follows that our measure of one quarter spells is a downward biased estimate of the number of spells that last less than one quarter of calendar time, and similarly, that our measure of three-plus quarter spells is an upward biased measure of the number of spells that last at least three quarters of calendar time. Because we will focus on how these measures have changed over time, we do not feel that this is a first order issue. In the theoretical analysis later in the paper, we solve the model at a weekly frequency and measure the three categories of spells in a way that parallels how they are measured in the data.

\subsection{Four Facts}

In this subsection we utilize the QWI data to document four pertinent facts regarding changes in the nature of labor market turnover that have occurred over the period 1999 to 2015. They are:

1. Labor market turnover, as measured by either the hires rate $h_{t}$ or the separations rate $s_{t}$, has decreased significantly. Changing composition of worker and firm characteristics accounts for only a small fraction of this aggregate decline.

2. A reduction in short-duration (one and two quarters) employment spells accounts for a very large fraction of the reduction in turnover. The decline in short-duration employment spells is pervasive. It has occurred across all age-sex groups, industries, and firm size categories.

3. The decline in the incidence of the shortest employment spells - ones that last less than a quarter - can be broken down into two components: a declining hires rate $h_{t}$ and a declining hazard rate for first-quarter spells, $\delta_{1 t}$. The two components each account for about half of the decline in the incidence of one quarter spells.

4. The hazard rates of employment spells of short tenure, $\delta_{1 t}$ and $\delta_{2 t}$, have declined by much more than the hazard rates of employment spells with longer tenure, $\delta_{3 t}$.

In the remainder of this subsection, we provide the evidence on each of these findings.

\subsubsection{The Decline in Labor Market Turnover}

The first fact that we document is a large decline in worker turnover. This fact has been documented elsewhere, often using other data sources (see, for example, Davis and Halti- 


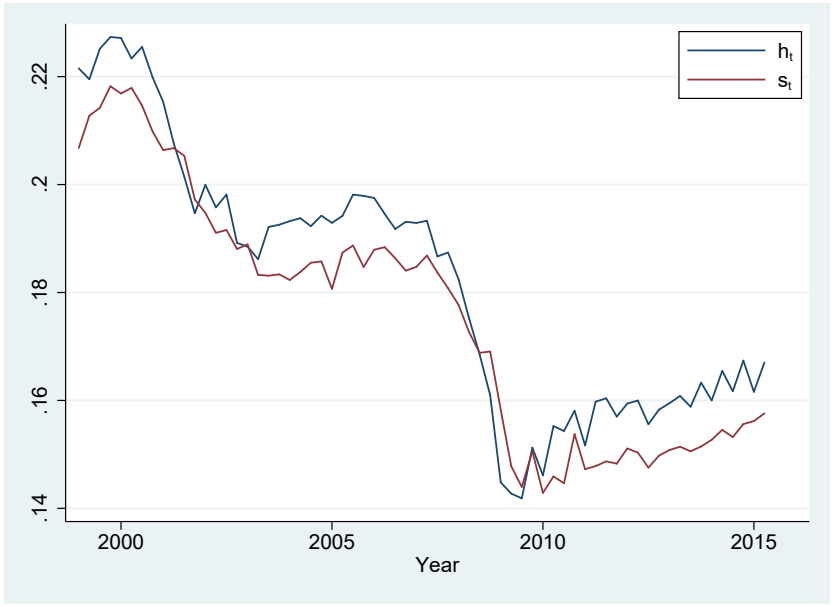

Figure 1: The hires rate and separations rate have declined significantly since 1999.

wanger (2014) and Hyatt and Spletzer (2013)). We document it here as it is important to have internally consistent measures from our data set.

Figure 1 displays the time series for both the hires rate, $h_{t}$, and the separations rate, $s_{t}$. It is clear from the figure that there has been a significant downward trend in labor market turnover over the sample period covered. The declines were greatest during the 2001 and 2007-8 recessions, and in each case there was minimal recovery of the two turnover measures subsequent to the recession. Comparing the rates in 2015 with the rates in 1999 (two "full employment" years), we see that both have declined by about $25 \%$.

It is well known that turnover rates vary with worker and firm characteristics, and this period has seen significant changes in the distributions of those characteristics. While the changing distributions of worker and firm characteristics can account for some of the aggregate decline just noted, it is important to note that the decline is observed even when controlling for these characteristics. Figure 2 shows the beginning of period (1999) and end of period (2015) hires rates, conditioning on different characteristics. In each of the plots, the various sub-groups consistently lie beneath the 45-degree line, indicating that the decline in hires rates has been pervasive (workers aged 14-18 are the one exception). The plots look very similar for separations rates and so are not shown.

\subsubsection{The Importance of Short-duration Employment Spells}

In order to assess the importance of separations among short-lived spells in the overall decline of the separations rate, we decompose the separations rate into the components accounted for by our three tenure categories.

Note that $Q_{1 t}, Q_{2 t}$, and $Q_{3 t}$ give the contribution of one quarter, two quarter, and 

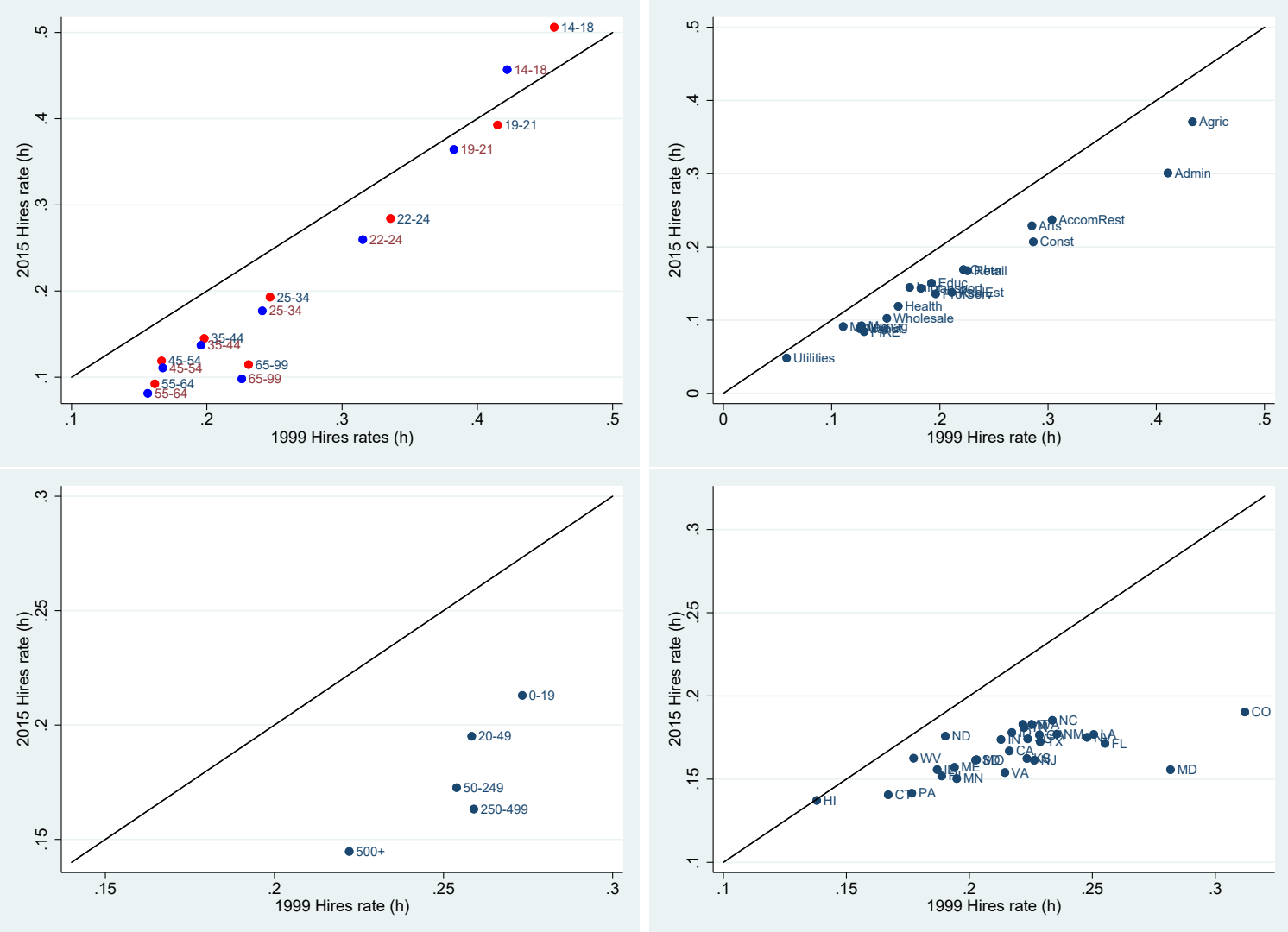

Figure 2: The panels in this figure plot, for various groupings of the data, the value of $h_{t}$ at the end of the sample (2015) against the value of $h_{t}$ at the beginning of the sample (1999). The upper left panel shows different age-sex groups, with the red dots representing females, and blue dots representing males. The other three panels break the data down by industry, by firm size, and by state.

three-plus quarter spells to overall separations, $S_{t}$. We can divide them by $N_{t}$ to get their contributions to the separations rate, $s_{t}$. Figure 3 shows the time series for the three components. It is clear that declining separations among one quarter spells accounts for the biggest part of the overall decline in the separations rate, followed by the contribution of two quarter spells. Declining separations among spells with three-plus quarters has contributed very little. Using the four-quarter average from 1999 as the initial value, and the fourquarter average from 2014Q3 to 2015Q2 (which we refer to as 2015) as the final value, $s_{t}$ declined by $26.9 \%$ from the beginning to the end of our sample. Of that overall decline, separations among one quarter spells accounted for $61.6 \%$, two quarter spells accounted for $24.4 \%$, and spells of three-plus quarters accounted for the remaining $14.0 \%$. Clearly, short-lived spells account for a very large fraction of the overall decline in turnover.

As with the overall decline in turnover, it is natural to ask whether the decline in 


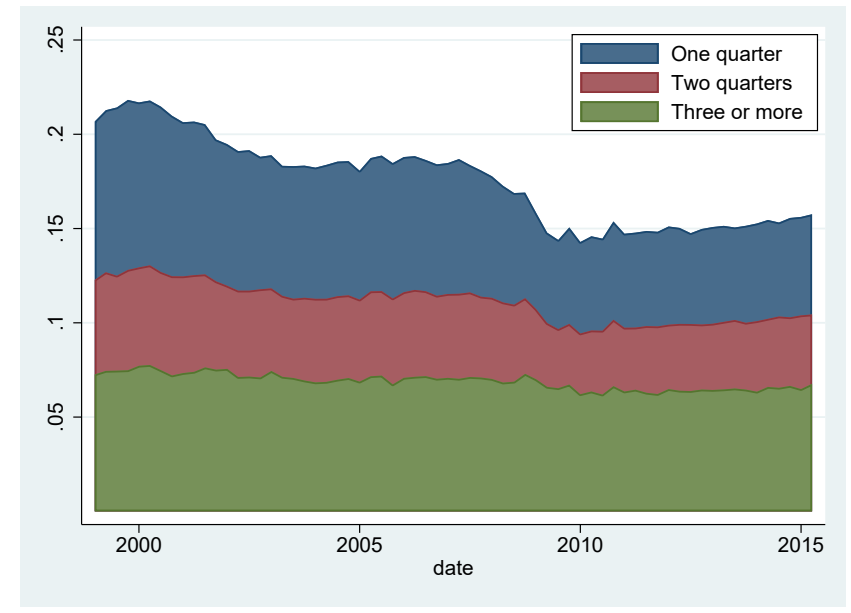

Figure 3: Decomposition of the separations rate $s_{t}$ into the parts accounted for by employment spells with one quarter, two quarter, and three-plus quarters of tenure.

short-duration employment spells is pervasive, or whether it is concentrated among workers or firms with particular characteristics. The answer is that the decline is pervasive: it has occurred among all types of workers and all types of firms. We demonstrate this by focusing on $q_{1 t}$, though the same patterns are also seen in $q_{2 t}$. Figure 4 plots, for various worker-level and firm-level groupings of the data, the value of $q_{1 t}$ at the end of the sample (2015) against its value at the beginning of the sample (1999). It is apparent from the upper-left plot of the figure that younger workers, and to a slightly lesser extent females, generally exhibit a higher $q_{1 t}$. More significantly, all of the age-sex groups lie well below the 45-degree line, indicating that the decline in $q_{1 t}$ occurred among all types of workers. It is also worth noting that the declines were roughly proportional, i.e. they were greater for the groups that initially had higher levels.

Hyatt and Spletzer (2017) show that the declines in $q_{1 t}$ experienced by different types of workers and different types of firms, as seen in the figures here, account for almost all of the overall decline in $q_{1 t}$; very little can be accounted for by composition effects (increases in worker types or firm types that have lower values of $q_{1 t}$ ).

\subsubsection{Decomposing the Decline in $q_{1 t}$}

While our focus is on the decline of short-duration employment spells generally, we have seen that the decline of one quarter spells has been particularly pronounced. All one quarter employment spells in quarter $t$ satisfy two conditions: they must have been initiated in quarter $t$ and must have been terminated during the same quarter. It follows that changes in the incidence of one quarter spells can be decomposed into changes in the hires rate and 

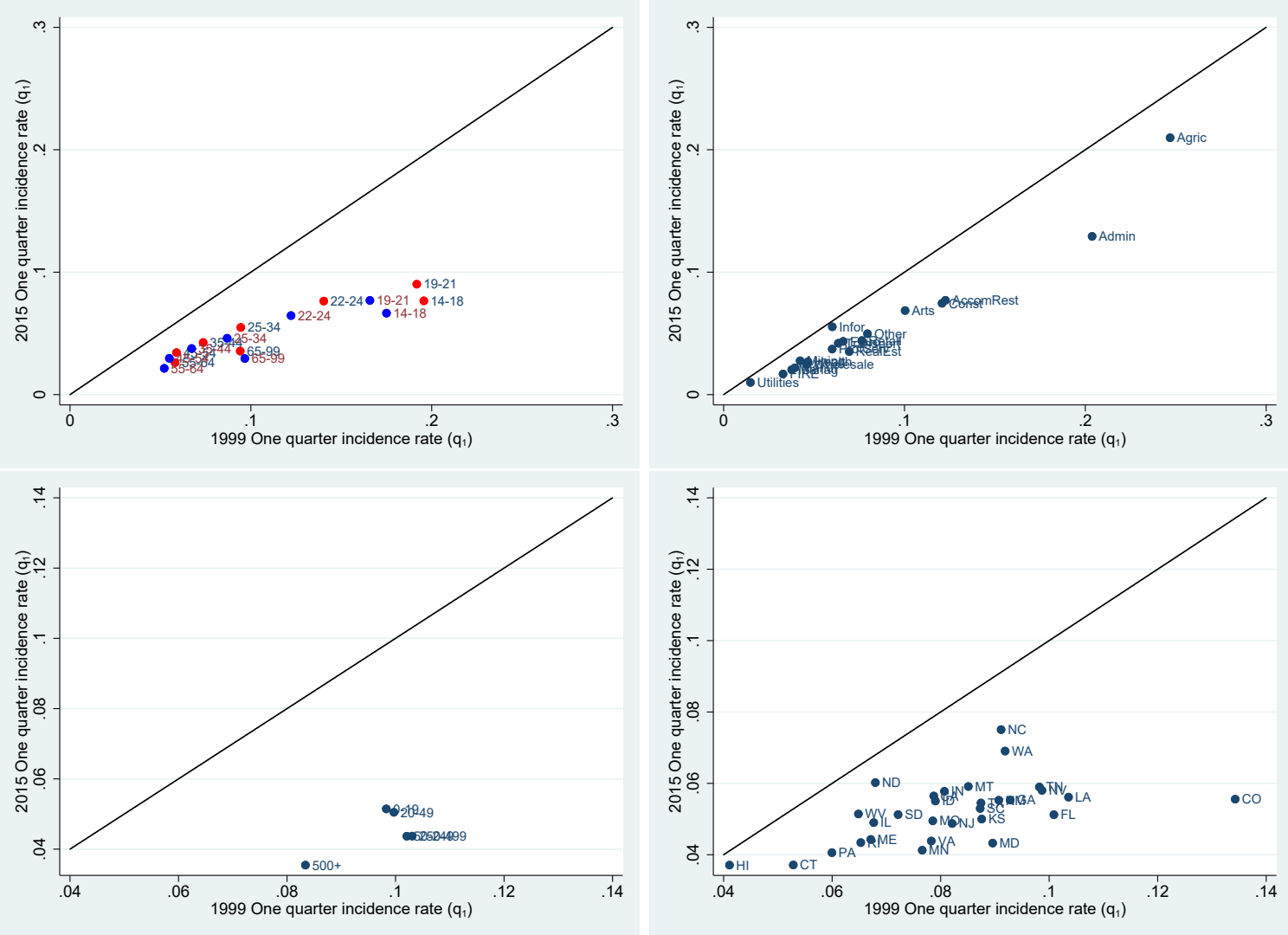

Figure 4: The panels in this figure plot, for various groupings of the data, the value of $q_{1 t}$ at the end of the sample (2015) against the value of $q_{1 t}$ at the beginning of the sample (1999). The upper left panel shows different age-sex groups, with the red dots representing females, and blue dots representing males. The upper right panel breaks the data down by industry, the lower left panel breaks it down by firm size, and the lower right panel by state.

changes in the hazard rate that a new hire does not survive into the next quarter. That is, $q_{1 t}=\frac{Q_{1 t}}{N_{t}}=\frac{Q_{1 t}}{N_{1 t}} \frac{N_{1 t}}{N_{t}}=\delta_{1 t} h_{t}$.

Figure 5 shows the time series for each of these two determinants of $q_{1 t}$. Again using the four-quarter averages for 1999 and 2014Q3-2015Q2 as the beginning and end periods to calculate the percentage declines in these two rates, the one quarter hazard rate declined by $19.1 \%$ ( 0.3901 to 0.3158$)$ and the hires rate declined by $26.4 \%(0.2234$ to 0.1644$)$. As such, the decline in the one quarter hazard rate accounted for a bit less than half of the overall decline in the incidence of one quarter spells. 

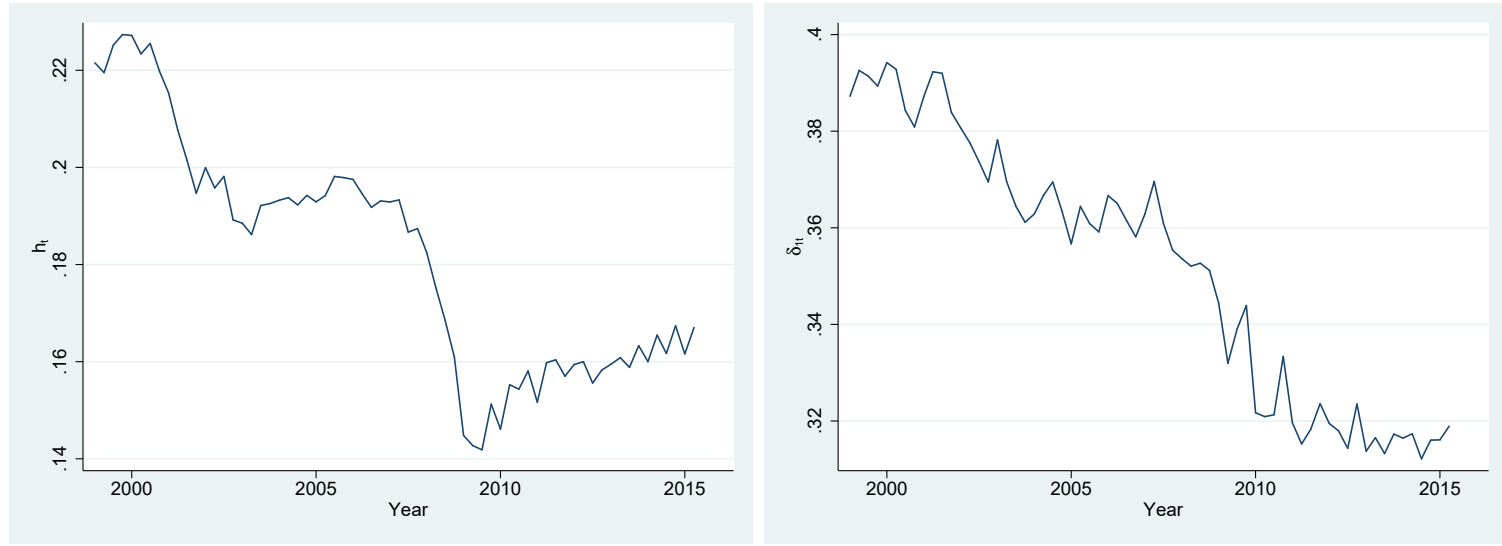

Figure 5: The left panel shows the hires rate, $h_{t}$, and the right panel shows the firstquarter hazard rate, $\delta_{1 t}$.

\subsubsection{Hazard Rates and Employment Spell Duration}

The previous subsection showed that the hazard rate for new employment spells has fallen considerably in the last two decades. This will obviously generate a decline in the overall separations rate, holding all else constant. It is of interest to know to what extent the hazard rates of two quarter and three-plus quarter spells have also declined.

The time series for the three hazard rates are displayed in Figure 6. The hazard rates for employment spells with one or two quarters of tenure are very similar to each other and are considerably higher than for employment spells with longer tenure. Moreover, between 1999 and 2015 the one quarter hazard rate and two quarter hazard rate declined by 0.0743 (0.3901 to 0.3158 ) and 0.0480 (0.3833 to 0.3353$)$, both of which are considerably greater than the decline in the three-plus quarter hazard rate, which was 0.0241 (0.1155 to 0.0914). ${ }^{5}$

\subsubsection{Summary}

The evidence presented here leads to a simple message. Any explanation for the large decline in worker turnover must involve an explanation for the large decline in the incidence of short-duration employment spells, and in particular the large decrease in the hazard rates of low-tenure spells.

\footnotetext{
${ }^{5}$ The percentage drop in the hazards are more similar, but as we will see later when we present our model, the level differences are informative about asymmetric effects across the tenure distribution.
} 


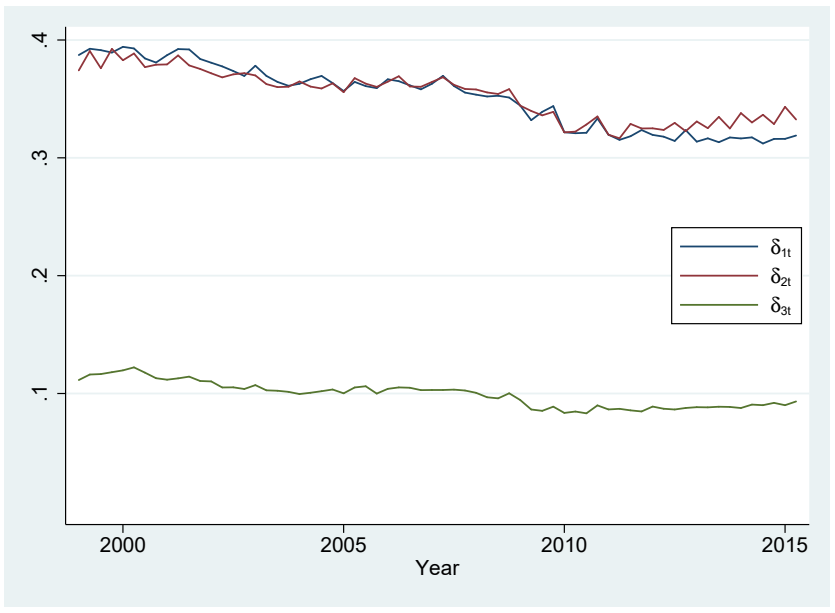

Figure 6: This figure shows the hazard rates for employment spells with one, two, and three-plus quarters of tenure.

\section{Potential Explanations}

Many potential explanations come to mind when trying to account for these features of the data. One possibility is that the manner in which firms fill vacant positions has changed over time, leading them to hire workers that are better matched and hence create matches that are more likely to persist. We will explore this possibility quantitatively in the model below. However, there are other plausible explanations as well. In this section we note four alternative potential explanations and argue that each of them seems unlikely or incomplete as an explanation for the observed changes.

\subsection{Decline in Seasonal Employment}

Seasonal employment naturally leads to short-duration employment spells. It follows that one natural hypothesis for the decreased incidence of short-duration employment spells is that the nature of seasonal changes in employment - summer jobs by young people, retail jobs around the holiday shopping season-have changed over time. While the data clearly show those seasonal elements - e.g. short-duration employment spells are especially common in the fourth quarter of the year - there does not appear to be any significant trend decline in these seasonal elements. Figure 7 shows time series for $q_{1 t}$ and $q_{2 t}$, both seasonally adjusted and not seasonally adjusted. The seasonal component of $q_{2 t}$ is especially high in the third quarter, as one would expect for summer jobs that begin in June and end in August. In both panels of the figure, we see that the seasonal element remains important throughout the entire period, and sits on top of an underlying downward trend in shortduration employment spells. 

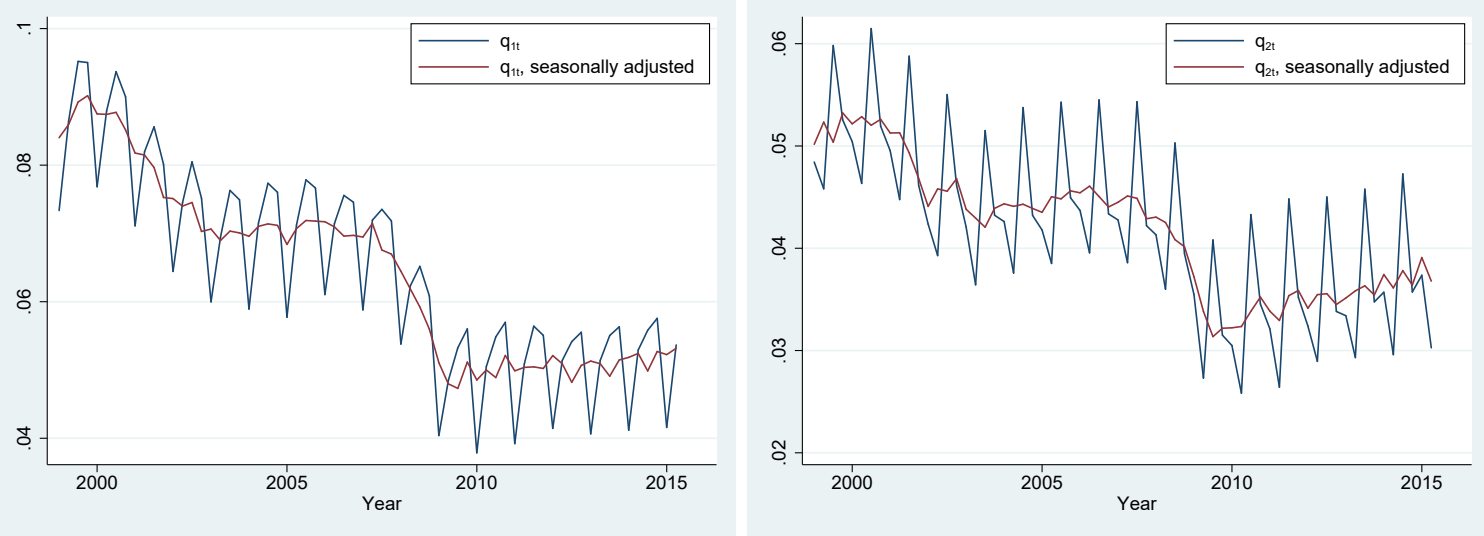

Figure 7: The left panel shows the seasonally adjusted and non-seasonally adjusted $q_{1 t}$. The right panel shows the adjusted and unadjusted $q_{2 t}$.

We conclude that a shrinking seasonal element does not explain the decline in shortduration employment spells.

\subsection{Decline in Temporary Layoffs and Recalls}

A second possible explanation is that the layoff/recall behavior of firms has changed. If firms hire workers for short periods, lay them off, and then subsequently recall them, this could generate short-duration employment spells. Moreover, if this sort of layoff/recall behavior declined between 1999 and 2015, then it could account for the observed decline in short-duration employment spells.

It is possible to assess this hypothesis with the QWI data. Specifically, the data allows us to condition on worker-employer matches which had no previous match within the last four quarters; conditioning in this way allows us to exclude most instances of the layoff/recall behavior (only recalls from matches that had existed more than four quarters prior would remain). In figure 8, we compare the hires rate among "new" matches (no employment in previous 4 quarters) with the overall hires rate (given the need for employment information from the previous 4 quarters, states whose coverage begins in 1999 do not have data for the conditional hires rate until 2000, thus both series are shown here for the period beginning in 2000). If much of the decline in short-duration spells could be attributed to a decline in layoff/recall behavior, then one would expect that the hires rate among "new" matches, which excludes these recalls, would not have declined by much. However, as figure 8 shows, the trend in the hires rate that conditions on the absence of earnings in the previous four quarters looks very much like the trend in the overall hires rate. As such, we conclude that changes in layoff/recall behavior by firms do not account for the declining labor market 


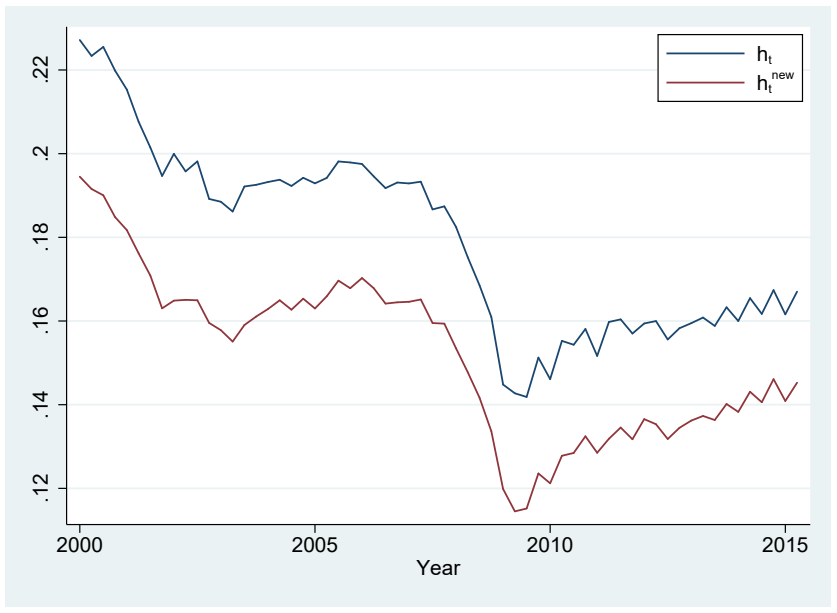

Figure 8: This figure compares the hires rate with a measure of the "new" hires rate that conditions on the absence of any earnings in the previous four quarters.

turnover that we observe. ${ }^{6}$

\subsection{Increased Outsourcing to Temp Agencies}

A third possibility is that firms have increasingly outsourced more of their short-term labor needs to temp agencies. That is, perhaps firms that used to bring on workers for shortduration employment spells to address brief spikes in labor demand have shifted toward utilizing temp agencies, and the workers at those temp agencies show up as continually employed by the temp agency, even though in reality they are stringing together brief stints at various firms.

If increasing reliance on temp agencies has displaced a significant number of short-term employment spells, we would expect to see that employment in temp agencies has risen over the 1999-2015 period. However, according to the ASA Staffing, Employment and Sales Survey American Staffing Association (2018), temporary and contracting staffing was 3.09 million in 1999, and 3.22 million in 2015. We conclude that outsourcing to temp agencies does not seem to explain the decline in short duration employment spells.

\subsection{Short-term Job Creation vs. Worker Churn}

A common element among each of the last three potential explanations is that they all reflect situations in which firms have temporary increases in labor demand. That is, they

\footnotetext{
${ }^{6}$ While the QWI data do not allow us to calculate the incidence of one quarter spells conditional on no earnings in the previous four quarters, it is nevertheless possible to calculate the incidence of one or two quarters spells conditional on no earnings in the previous four quarters. This measure of short-term employment spells also shows no difference in trends between the conditional and unconditional series.
} 
all describe situations in which short-duration employment spells reflect short-duration jobs.

While we have argued that none of the previous three explanations seems to provide a promising explanation for the decline in short-duration employment spells, it is still nevertheless possible that firms have reduced their tendency to create short duration jobs (in ways unrelated to seasonal hiring, recalls, or temp agencies) as a way to deal with temporary spikes in labor demand. That is, short-duration employment spells occur either because firms churn through workers in existing jobs or because firms create short-duration jobs, and we want to assess whether the decline in short-duration employment spells is associated with a decline in short-duration job creation, or whether it is more related to a decrease in worker churn within existing jobs.

Identifying the extent to which the decline in short-duration employment spells has resulted from a reduction in worker churn within existing jobs versus a decline in shortduration job creation is an important diagnostic to help us better understand the forces responsible for the decline in short-duration employment spells. If a decline in short-duration job creation is a dominant source of the overall decline, it suggests either that firms now face fewer short-term fluctuations in demand or that they have moved away from an employment strategy which deals with short-term fluctuations in their labor needs by adding and reducing workers, toward one by which they would deal with the short-terms labor needs by some other means - longer hours, increased worker effort, etc. On the other hand, if reduced worker churn within existing jobs has been a dominant source of the decline, it suggests that firms have improved their ability to recruit workers who the firm will want to, and be able to, retain.

The QWI provides data on job creation and job destruction, defined in the standard way as changes in firm-level employment, aggregated over firms that are growing (job creation) and over firms that are shrinking (job destruction). In order to determine whether firm employment has grown or declined, one must compare employment at two points in timethe end of one quarter relative to the end of the previous quarter. A worker is considered employed at the end of a quarter if he or she receives earnings in that quarter and in the subsequent quarter. A worker's employment can therefore only contribute to the QWI job creation measures if that worker receives earnings in two consecutive quarters. As a result, one quarter employment spells as we have defined them-earnings in the initial quarter of employment, but not in the subsequent quarter of employment - by construction cannot show up in the job creation measures. Nevertheless, we can still gain insight into the reduction in short-duration employment spells by focusing on the spells that last two quarters, and examining whether they have tended to decline more in the same places that job creation has declined.

Workers who are hired into employment spells that last through the end of the initial 
quarter can be divided into (1) hires that constitute job creation (hired into a new job at an expanding firm) and (2) hires that constitute "worker churn" (hired to replace a departed worker, either at a growing firm or at a shrinking firm). Ideally, we would observe this breakdown on a firm-by-firm basis, so that we could assess whether the short-duration employment spells (and their decline over time) are more associated with short-term job creation, or with worker churn.

While this is not possible with the QWI public use data, which aggregates over firms, we can still examine the question at the various subcategories of aggregation that are available in the QWI. We do that here, using industry-state cells as the unit of observation. Importantly, there is substantial variation across these industry-state cells in the magnitude of the decline in short-duration employment spells. Looking across the 570 industry-state combinations (30 state, 19 industries), we then examine the extent to which the declines in short-duration employment spells are more closely related with declines in job creation or with declines in worker churn.

To begin, we show that short-duration employment spells are indeed much more prevalent in industry-state cells where either job creation is high or where worker churn is high. ${ }^{7}$ Figure 9 shows the relationship between the incidence of two quarter employment spells and worker churn in the top two plots and between the incidence of two quarter employment spells and the job creation rate (job creation divided by total employment spells) in the middle two plots, for 19 industries in 30 states. The bottom two plots show the relationship between worker churn and job creation. The plots on the left show the beginning of the sample (1999) and the plots on the right show the end of the sample (2015).

It is clear that short-duration spells are more prevalent in the industry-state cells where worker churn is higher and where job creation is higher (positive slopes in the upper and middle panels). It is also notable that the slopes of the relationships in figure 9 change minimally between the beginning of the sample and the end of the sample. Thus, the reductions in labor market turnover that occurred between the beginning of the sample and the end of the sample took the form of a downward/leftward shift in the figures, along relatively stable slope-lines.

Of course, it is not surprising that two quarter employment spells would be more common in industry-state cells where worker churn was higher and where job creation was higher. What is of greater interest, however, is to see whether the decline in two quarter employment spells was more pronounced in industry-state cells where job creation declined by more, or

\footnotetext{
${ }^{7}$ Again, we use employment spells that last two quarters, rather than one quarter employment spells, as our measure of short-duration employment spells, given that the job creation and worker churn measures only include employment spells that last through at least the end of their first quarter. Nevertheless, the incidence of employment spells that last two quarters is highly correlated (across industry-state cells) with the incidence of employment spells that last only one quarter.
} 

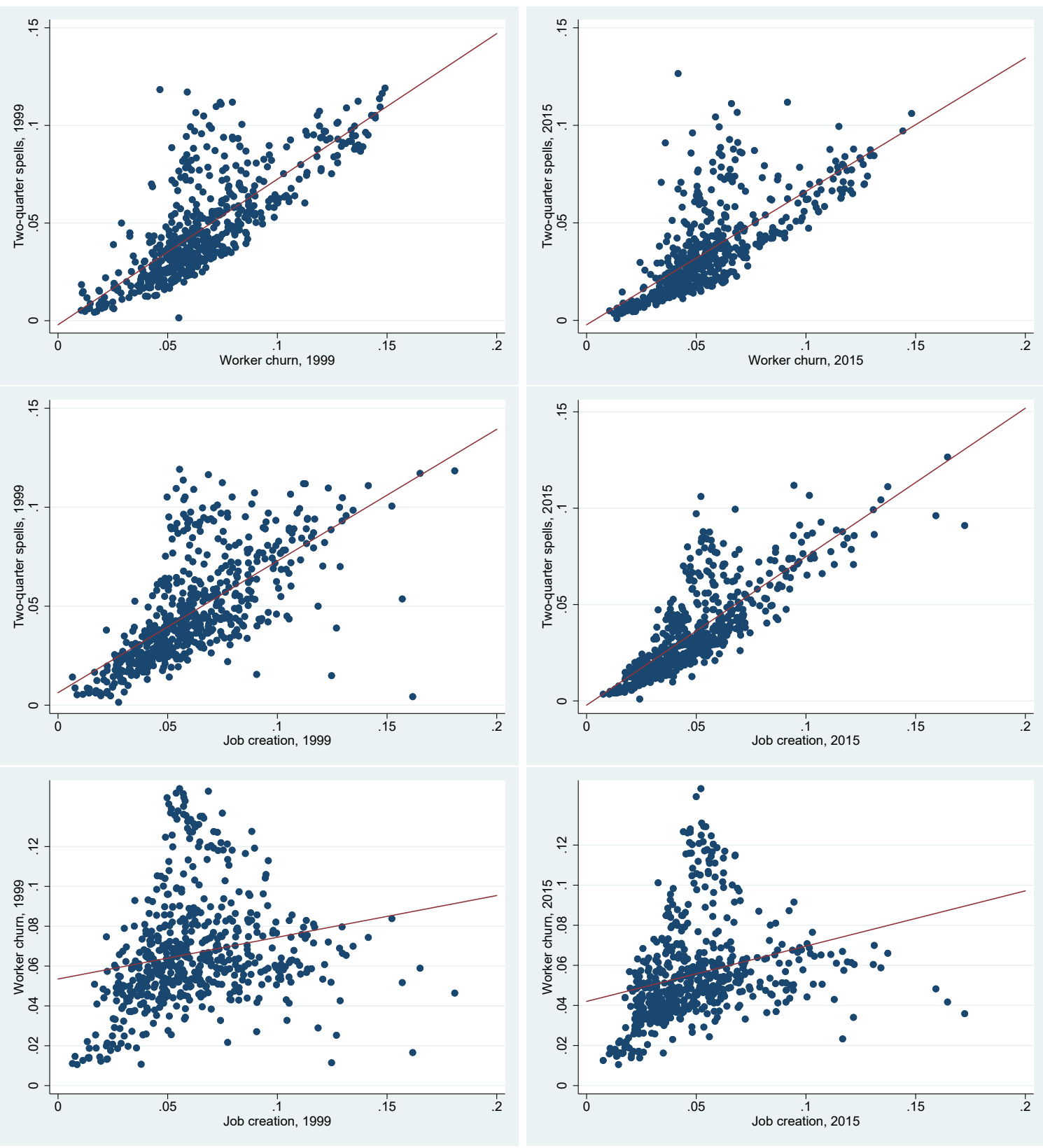

Figure 9: The top two plots show the relationship between the incidence of two quarter spells, $q_{2 t}$, and worker churn, with the figure on the left showing the relationship at the beginning of the sample, and the figure on the right showing the end of the sample. The middle two plots show the relationship between the incidence of two quarter spells and job creation rates, with the beginning of sample and end of sample on the left and right, respectively. The bottom two plots show the relationship between worker churn and job creation. The lines in the plots are regression lines. 

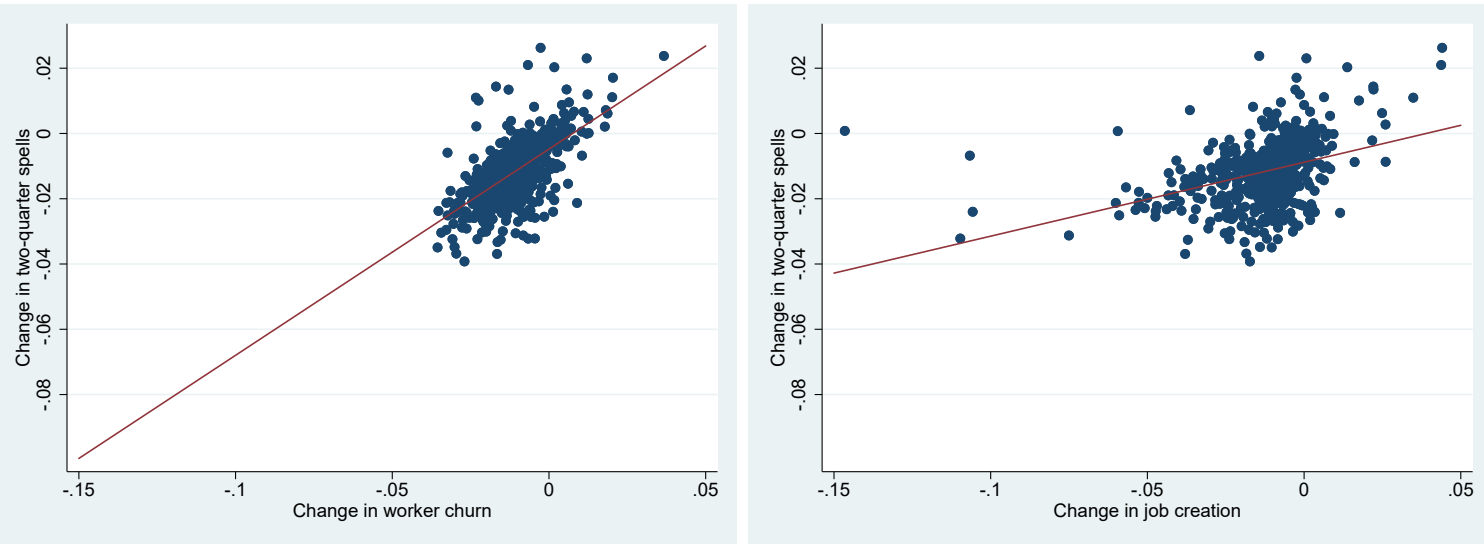

Figure 10: The left plot shows the relationship between the change in the incidence of two quarter spells, $q_{2 t}$, and the change in the rate of worker churn. The right plot shows the relationship between the change in $q_{2 t}$ and the change in the rate of job creation.

in industry-state cells where worker churn declined more. That is, was the decline in shortduration jobs more associated with a drop in worker churn, or with a drop in job creation?

Figure 10 helps to answer that question. It shows the relationship between the change (from 1999 to 2015) in the incidence of two quarter spells and (1) the change in worker churn (in the left plot) and (2) the change in the job creation rate (in the right plot). As is clear from the figure (and the regression lines in each plot), changes in short-duration spells was more correlated with changes in worker churn. Note that if all of the decline in two quarter spells for a given industry-state cell resulted from a decline in short-term job creation, we would observe no change in worker churn and a drop in two quarter spells for that industry-state cell. Instead, we see in the left panel of the figure that reductions in two quarter spells are closely linked to reductions in worker churn. On the other hand, if all of the decline in two quarter spells resulted from a decline in worker churn, we would see reductions in two quarter spells with no drops in job creation. In the right panel, we do see some modest association of declining two quarter spells with declining job creation.

The cross-sectional relationships between (changes in) the rate of two quarter employment spells and (changes in) worker churn and job creation are shown in the regression results in Table $1 .^{8}$ A couple of observations deserve comment. First, changes in worker churn are more strongly associated with changes in short-duration spells than are changes in job creation, as indicated by the larger regression coefficient and the higher $R^{2}$. Second, including job creation in the regression that already has worker churn in it does not really change the coefficient on worker churn. This result occurs because the changes in job

\footnotetext{
${ }^{8}$ If we use changes in the job destruction rate, rather than changes in the job creation rate, we get very similar results. In the interest of parsimony, we just report the job creation results.
} 
Table 1: Regression results for industry-state cross section

\begin{tabular}{lccc}
\hline \hline & \multicolumn{3}{c}{ Change in incidence of two quarter spells } \\
\hline constant & -0.0088 & -0.0048 & -0.0015 \\
& $(0.0005)$ & $(0.0005)$ & $(0.0005)$ \\
$\Delta$ jc & 0.2265 & & 0.2477 \\
& $(0.0232)$ & & $(0.0166)$ \\
$\Delta$ churn & & 0.6320 & 0.6551 \\
& & $(0.0333)$ & $(0.0283)$ \\
\hline$R^{2}$ & 0.1441 & 0.3891 & 0.5609
\end{tabular}

Notes: Regression results for a cross-section of 570 industry-state cells. Changes (between 1999 and 2015) in the incidence of two quarter employment spells are regressed on changes in the job creation rate and changes in the worker churn rate. Standard errors appear in parenthesis.

creation and in worker churn are essentially uncorrelated. In a regression of the change in worker churn on the change in job creation, the coefficient is -0.0319 and it is not statistically significant. This result might seem somewhat surprising, as one might think that there is always going to be some churn associated with filling newly created jobs, and as a result worker churn would rise or fall proportionately with job creation. This does not appear to be the case. Loosely speaking, high rates of job creation do not seem to give rise to higher levels of worker churn associated with the newly created jobs.

While not definitive, these results suggest that a large part of the decline in short-term employment spells is associated with a decline in worker churn, and so motivate us to consider a model that focuses on the role of hiring decisions in shaping subsequent worker turnover.

\section{A Model of Employment Spells}

In this section, we describe the model that we will use to provide some insights into the driving forces behind the patterns documented in the previous sections. The model incorporates learning about match quality into an otherwise standard Mortensen-Pissarides (1994) framework. ${ }^{9}$ We show that in steady state equilibrium the model implies a simple stochastic process for employment spell dynamics that is completely characterized by three parameters. The model could be extended along various dimensions without affecting this characterization of the process for employment spell dynamics, but we focus on the simplest

\footnotetext{
${ }^{9}$ The model is similar to our model in Pries and Rogerson (2005), but our focus here is on understanding changes in the U.S. labor market, whereas our focus in that paper was on cross-country differences. Relative to Pries and Rogerson (2005), here we explicitly model how the initial perceived probability that a match is good relates to the noisiness of the initial productivity signal.
} 
version to facilitate exposition.

\subsection{Model}

Time is discrete. There are two types of agents: workers and entrepreneurs. There is a unit measure of workers, each with preferences over consumption and leisure given by

$$
\sum_{t=0}^{\infty} \beta^{t}\left[c_{t}-b h_{t}\right]
$$

where $0<\beta<1$ is the discount factor, $b>0$ is the disutility associated with working, $c_{t} \geq 0$ is consumption in period $t$, and $h_{t} \in\{0,1\}$ is time devoted to working in period $t$. There is a much larger measure of entrepreneurs. Each entrepreneur has the ability to costlessly create a production opportunity that can in turn produce output using exactly one worker. ${ }^{10}$ After creating a production opportunity, an entrepreneur bears a recruiting cost $k_{r}$ per period while searching for a worker, measured in units of utility. We follow the literature in referring to the act of incurring the recruiting cost as "posting a vacancy." Each entrepreneur has preferences given by:

$$
\sum_{t=0}^{\infty} \beta^{t}\left[c_{t}-I_{t}^{r} k_{r}\right]
$$

where $I_{t}^{r}$ is an indicator function for whether the entrepreneur incurs a recruiting cost in period $t$. In what follows we will refer to an entrepreneur that has created a production opportunity as a firm.

Unemployed workers and firms are brought together by a standard constant-returns-toscale matching technology, with the number of meetings each period given by $m(u, v)$ where $u$ is the number of unemployed workers and $v$ is the number of vacancies. The rate at which workers meet firms is given by $p_{w}(\theta)=m(u, v) / u=m(1, \theta)$, where $\theta$ is the $v / u$ ratio. The rate at which firms meet unemployed workers is given by $p_{f}(\theta)=m(u, v) / v=m(1 / \theta, 1)$.

When an unemployed worker and a firm meet, they must decide whether to form an employment relationship. The productivity of a match, $y$, can equal one of two values: $y \in\left\{y_{b}, y_{g}\right\}$, with $y_{g}>y_{b}$. However, $y$ is not observed at the time of the meeting. Instead, the worker and firm observe the same noisy "inspection" signal $z$, where $z=y+\epsilon$ and $\epsilon \sim N\left(0, \sigma_{\epsilon}\right)$. The prior probability that the match is good (i.e. $y=y_{g}$ ) is given by $\pi_{0}$. After observing $z$, the worker and firm each calculate the posterior probability that they

\footnotetext{
${ }^{10}$ As is well known, this is without loss of generality in the current context. One can abstract from having agents identified as entrepreneurs and instead assume a holding company makes all decisions about job creation and vacancy posting, with ownership of the holding company distributed among the workers.
} 
have a good match:

$$
\pi(z)=\frac{\pi_{0} f\left(z-y_{g}\right)}{\pi_{0} f\left(z-y_{g}\right)+\left(1-\pi_{0}\right) f\left(z-y_{b}\right)}
$$

where $f(\epsilon)$ is the normal density. They base their match formation decision on this posterior $\pi(z)$ (which, going forward, we denote simply by $\pi$ since the value of $z$ is of no independent relevance). This noise structure implies a distribution for the posterior, whose cdf we denote by $H(\pi)$. If the noise $\sigma_{\epsilon}$ is very small, so that a lot is learned from the initial signal, then the density of this posterior will have a mass of approximately $1-\pi_{0}$ that is bunched around zero and another mass of approximately $\pi_{0}$ that is bunched around 1 . The greater the noise $\left(\sigma_{\epsilon}\right)$, the more the density will concentrate around $\pi_{0}$. In order for the match type to be of substantive importance, we will assume that $y_{b}$ is sufficiently low that matches known to be bad will be dissolved, and that matches known to be good will not be dissolved.

In what follows we will treat the value of $\sigma_{\epsilon}$ as an exogenous parameter and characterize equilibrium taking its value as given. More generally, we think of this parameter as reflecting a choice of the firm regarding its recruiting and screening practices. Although the firm would prefer a lower value of $\sigma_{\epsilon}$ to a higher value, this requires a more costly screening process and so the optimal choice of the firm reflects a trade-off of costs and benefits. Changes in technology that affect the costs of achieving different values of $\sigma_{\epsilon}$ would then influence the firm's optimal choice. Given this new value for $\sigma_{\epsilon}$, we can use our model to assess the consequences. Implicitly, there is a configuration of screening costs that make a particular value of $\sigma_{\epsilon}$ the optimal choice. We treat the underlying costs as a latent variable and focus purely on the effects of $\sigma_{\epsilon}$.

If the worker and the firm decide to form a match, a start-up cost $k_{h}$ is incurred in the first period of the match. This cost includes the costs of training a new worker and/or the administrative costs associated with getting a new worker set up. We assume for simplicity that the cost is incurred only in the initial period. We could instead assume that the startup costs are paid for each of several periods at the beginning of the match without affecting the key results. ${ }^{11}$

At the beginning of each period after a match's initial period, the worker and firm learn the match's type with probability $\alpha$. This "all-or-nothing" learning can be understood as the outcome of another noisy signal, the noisy component of which is uniformly distributed, so that sufficiently high signals reveal that the match is good, sufficiently low signals reveal that the match is bad, and signals in between do not change the prior probability $\pi$ that the match is good. Alternatively, we could assume that the noise in subsequent signals is normally

\footnotetext{
${ }^{11}$ We could also accommodate a dismissal cost that needs to be paid in the event that the match is dissolved for any reason other than the worker choosing to separate for exogenous reasons. With Nash bargaining, this acts similar to a start-up cost except that it is the discounted expected present value of the cost that matters.
} 
distributed just like the initial signal, so that the worker and firm would continuously update their prior probabilities that the match is good, but we opt for the uniform distribution because it allows for the simple characterization of employment spell dynamics that we derive below. ${ }^{12}$

In addition to allowing matches to endogenously dissolve when they are revealed to be bad, we also allow for an exogenous source of separations. In particular, at the beginning of each period, and independently of the underlying match quality, the match will dissolve with probability $\lambda^{13}$

The wage paid by a firm to a worker will be determined via Nash bargaining, with $\eta$ denoting the worker's bargaining power. This Nash wage will depend on the probability that the match is good, as well as whether the match is new or continuing (new matches differ from continuing matches because the cost $k_{h}$ is only incurred in the initial period). We will denote the wage payments in new and continuing matches by $w^{n}(\pi)$ and $w^{c}(\pi)$, respectively. Nash bargaining implies that workers and firms will always agree on match formation and dissolution decisions. Because we assume that matches known to be good always produce and matches known to bad will always be dissolved, match formation decisions will take the form of a threshold rule. That is, only matches with a sufficiently high value of $\pi$ will not be formed.

We close this section by noting one feature implicit in our model specification, which is that we do not allow for on-the-job search. This assumption serves to simplify the analysis. But more importantly, given our focus on short duration employment spells, we note that Hyatt and Spletzer (2017) provide evidence that the dominant transitions for these short duration spells are transitions into and out of unemployment as opposed to transitions from job to job. Having said this, we note that in our empirical analysis we will assume that a period corresponds to a week, and for standard time aggregation reasons it follows that we would necessarily observe some job-to-job transitions at a monthly or quarterly frequency of observation, so the absence of on-the-job search in the model is not equivalent to ruling out what would appear to be job-to-job transitions in monthly or quarterly data.

\footnotetext{
${ }^{12}$ We do not think that normally distributed noise would give rise to substantive differences. Qualitatively, the biggest change is that match type would never be revealed with certainty, and separation rules for continuing matches would involve a tenure specific threshold rule. This would have a small impact on the match tenure distribution for higher tenure levels, but since our focus is on short tenure spells, we do not think this would be significant for our quantitative results.

${ }^{13}$ In principle, one could break this exogenous separation rate into two pieces, one reflecting exogenous job destruction and the other reflecting exogenous worker separations. Because our analysis does not distinguish between worker and job flows there is no need to distinguish between exogenous separations in which the job is destroyed and separations in which the worker and firm separate but the firm hires a new worker into the position.
} 


\subsection{Steady State Equilibrium}

We study the steady state equilibrium for the model just described. In the steady state, the unemployment rate is constant and so is the distribution of employment matches across values of $\pi$, the prior probability that the match is good.

We begin by developing the Bellman equations that describe the optimal decisions of workers and firms. The value to a firm of having a new match opportunity that is good with probability $\pi$ is

$$
\begin{aligned}
J^{n}(\pi)=\max \{ & \pi y_{g}+(1-\pi) y_{b}-w^{n}(\pi)-k_{h} \\
& \left.+\beta(1-\lambda)\left[\alpha \pi J^{c}(1)+\alpha(1-\pi) J^{c}(0)+(1-\alpha) J^{c}(\pi)\right]+\beta \lambda V, V\right\}
\end{aligned}
$$

where $V$ is the value of an unfilled vacancy and $J^{c}(\pi)$ is the value to a firm of a continuing match (which means the start-up cost $k_{h}$ has already been incurred). With probability $1-\lambda$, there is no exogenous separation, and with probability $\alpha$, the match's type is revealed. $J^{c}(1)$ denotes that the value of a match that is known to have productivity $y_{g}$, whereas $J^{c}(0)$ is the value of a match that is known to have productivity $y_{b}$.

The Bellman equation for a continuing match is given by:

$$
\begin{aligned}
J^{c}(\pi)=\max \{ & \pi y_{g}+(1-\pi) y_{b}-w^{c}(\pi) \\
& \left.+\beta(1-\lambda)\left[\alpha \pi J^{c}(1)+\alpha(1-\pi) J^{c}(0)+(1-\alpha) J^{c}(\pi)\right]+\beta \lambda V, V\right\}
\end{aligned}
$$

The firm's value from a vacancy satisfies

$$
V=-k_{r}+\beta\left[p_{f} \int_{0}^{1} J^{n}(\pi) d H(\pi)+\left(1-p_{f}\right) V\right]
$$

The value to a worker of a new match that is good with probability $\pi$ is

$$
E^{n}(\pi)=\max \left\{w^{n}(\pi)+\beta(1-\lambda)\left[\alpha \pi E^{c}(1)+\alpha(1-\pi) E^{c}(0)+(1-\alpha) E^{c}(\pi)\right]+\beta \lambda U, U\right\} .
$$

Similarly, the value to a worker of a continuing match that is good with probability $\pi$ is

$$
\left.E^{c}(\pi)=\max \left\{w^{c}(\pi)+\beta(1-\lambda)\left[\alpha \pi E^{c}(1)+\alpha(1-\pi) E^{c}(0)+(1-\alpha) E^{c}(\pi)\right]+\beta \lambda U\right), U\right\} .
$$

This only differs relative to $E^{n}(\pi)$ due to the wage $w^{c}(\pi)$, which will be higher than $w^{n}(\pi)$ since the current net output is higher. 
The worker's value from being unemployed is

$$
U=b+\beta\left[p_{e} \int_{0}^{1} E^{n}(\pi) d H(\pi)+\left(1-p_{e}\right) U\right]
$$

where $b$ is the flow utility of unemployment.

Under Nash bargaining, the worker and firm choose to produce so long as the match surplus is positive. Let the match surplus of a new match that is good with probability $\pi$ be given by $S^{n}(\pi)=J^{n}(\pi)+E^{n}(\pi)-U-V$, and the match surplus of a continuing match be given by $S^{c}(\pi)=J^{c}(\pi)+E^{c}(\pi)-U-V$. Using the above equations to substitute into these definitions, we have:

$$
\begin{aligned}
S^{n}(\pi)=\max \{ & \pi y_{g}+(1-\pi) y_{b}-k_{h}+\beta(1-\lambda)\left[\alpha \pi S^{c}(1)+\alpha(1-\pi) S^{c}(0)+(1-\alpha) S^{c}(\pi)\right] \\
& +\beta(U+V), U+V\}-U-V
\end{aligned}
$$

or

$$
\begin{aligned}
S^{n}(\pi)=\max \{ & \pi y_{g}+(1-\pi) y_{b}-k_{h}+\beta(1-\lambda)\left[\alpha \pi S^{c}(1)+\alpha(1-\pi) S^{c}(0)+(1-\alpha) S^{c}(\pi)\right] \\
& -(1-\beta)(U+V), 0\}
\end{aligned}
$$

Similarly,

$$
\begin{aligned}
S^{c}(\pi)=\max \{ & \pi y_{g}+(1-\pi) y_{b}+\beta(1-\lambda)\left[\alpha \pi S^{c}(1)+\alpha(1-\pi) S^{c}(0)+(1-\alpha) S^{c}(\pi)\right] \\
& -(1-\beta)(U+V), 0\}
\end{aligned}
$$

Free entry by firms ensures that in equilibrium $V=0$. From (6), we also have

$$
(1-\beta) U=b+\beta \eta p_{e} \int_{0}^{1} S^{n}(\pi) d H(\pi)
$$

Substituting these into the surplus equations, we have

$$
\begin{aligned}
S^{n}(\pi)=\max \{ & \pi y_{g}+(1-\pi) y_{b}-b-k_{h}+\beta(1-\lambda)\left[\alpha \pi S^{c}(1)+\alpha(1-\pi) S^{c}(0)+(1-\alpha) S^{c}(\pi)\right] \\
& \left.-\beta \eta p_{e} \int_{0}^{1} S^{n}(\pi) d H(\pi), 0\right\}
\end{aligned}
$$


and

$$
\begin{aligned}
S^{c}(\pi)=\max \{ & \pi y_{g}+(1-\pi) y_{b}-b+\beta(1-\lambda)\left[\alpha \pi S^{c}(1)+\alpha(1-\pi) S^{c}(0)+(1-\alpha) S^{c}(\pi)\right] \\
& \left.-\beta \eta p_{e} \int_{0}^{1} S^{n}(\pi) d H(\pi), 0\right\}
\end{aligned}
$$

The first arguments in the max operators of both $S^{n}(\pi)$ and $S^{c}(\pi)$ are linear and increasing in $\pi$. Thus, because we assume that $y_{b}$ is low enough that matches known to be of low productivity are not viable (i.e. $S^{n}(0)=0$ ), but $y_{g}$ is high enough that matches known to be of high productivity are viable $\left(S^{n}(1)>0\right)$, the match formation decision will take the form of a threshold rule, with threshold value $0<\pi^{n}<1$ that satisfies $S\left(\pi^{n}\right)=0$. Moreover, because the first argument in the max operator of $S^{c}(\pi)$ exceeds its counterpart in the equation for $S^{n}(\pi)$ by $k_{h}$, it is apparent that the threshold value $\pi^{c}$, defined by $S^{c}\left(\pi^{c}\right)=0$, is less than $\pi^{n}$ (and $\pi^{n}=\pi^{c}$ if $k_{h}=0$ ). Thus, once a match is formed, it continues to produce until either the match is discovered to be low productivity, or there is an exogenous separation.

The Nash wages can be found by imposing the sharing conditions $E^{c}(\pi)-U=\eta S^{c}(\pi)$ and $E^{n}(\pi)-U=\eta S^{n}(\pi)$ and substituting the Bellman equations above. The resulting wage equations are:

$$
\begin{aligned}
& w^{n}(\pi)=b+\eta\left[\pi y_{g}+(1-\pi) y_{b}-k_{h}-b+k_{r} \theta\right] \\
& w^{c}(\pi)=b+\eta\left[\pi y_{g}+(1-\pi) y_{b}-b+k_{r} \theta\right]
\end{aligned}
$$

That is, the worker earns what he or she would earn while unemployed, $b$, plus the share $\eta$ of the match's flow surplus. Note that the wage of a new match $w^{n}(\pi)$ is lowered by the presence of $k_{h}$ as the worker effectively shares the burden of the start-up cost with the firm.

We can now define an equilibrium of the model:

Definition 1. A stationary equilibrium is a set of values $\left\{\pi^{n}, U, V, u, \theta\right\}$ and a set of functions $\left\{J^{c}(\pi), J^{n}(\pi), E^{c}(\pi), E^{n}(\pi), S^{c}(\pi), S^{n}(\pi), w^{c}(\pi), w^{n}(\pi)\right\}$ such that:

1. Bellman equations: $J^{n}(\pi), J^{c}(\pi), V, E^{n}(\pi), E^{c}(\pi), U, S^{n}(\pi)$, and $S^{c}(\pi)$ satisfy $(1)$, (2), (3), (4), (5), (6), (7), and (8).

2. free entry: $\theta$ achieves $V=0$.

3. Nash bargaining: $w^{c}(\pi)$ and $w^{n}(\pi)$ satisfy the Nash bargaining conditions $E^{c}(\pi)-U=$ $\eta S^{c}(\pi)$ and $E^{n}(\pi)-U=\eta S^{n}(\pi)$.

4. optimal match formation: $\pi^{n}$ satisfies $S^{n}\left(\pi^{n}\right)=0$.

5. stationarity: The flows into and out of unemployment are equal. 
We note that solving for an equilibrium computationally is straightforward. Given an initial guess for $\theta$, the surplus functions can be found via value function iteration. One can then solve for the value $V$ given the surplus function. $V$ can easily be shown to be decreasing in $\theta$, so the guess of $\theta$ is adjusted appropriately depending upon whether $V>0$ or $V<0$, until $V=0$. Given the equilibrium value of $\theta$, the surplus function $S^{n}(\pi)$ is used to find the value $\pi^{n}$ that satisfies $S^{n}\left(\pi^{n}\right)=0$. The values for $\pi^{n}$ and $p_{e}(\theta)$ allow us to find the steady state equilibrium unemployment rate $u$ that satisfies the stationarity condition. Finally, the wage functions are easily calculated from (9) and (10) given the equilibrium $\theta$.

\subsection{Reduced Form Implications for Employment Spell Dynamics}

From the perspective of understanding the model's implications for the employment spell statistics from the QWI that we documented earlier, it turns out that the only equilibrium object that one needs to know is the threshold value $\pi^{n} .{ }^{14}$ Given this value and the model primitives, one can compute the probability that a match is good conditional on being formed, which we denote by $\pi^{g}:^{15}$

$$
\pi^{g}=\int_{\pi^{n}}^{1} \pi d H(\pi)
$$

Given $\pi^{g}$, along with the learning parameter $\alpha$ and the exogenous separation rate $\lambda$, we can solve for all of the statistics that we analyzed in section 2 . When connecting model outcomes to statistics derived from the QWI it is important to understand that the QWI involves a certain amount of time aggregation. For example, the measure of total employment spells in the QWI is not the number of spells at a point in time, but rather the total quantity of spells over the course of an entire quarter. In what follows, we will interpret the period length in the model to be one week, and describe how to compute the model implied counterparts for the QWI statistics of interest, using only the three values for $\pi^{g}, \alpha$, and $\lambda$.

Let $e^{j}(a)$ be the fraction of a given cohort of entrants that is of type $j \in\{g, b\}$ and is still employed through $a$ periods. ${ }^{16}$ By construction, $e^{b}(1)=\left(1-\pi^{g}\right)$, and $e^{g}(1)=\pi^{g}$. A simple iterative procedure can be used to compute the $e^{j}(a)$ for $a>1$. Specifically, because good matches are only destroyed when the match dissolves exogenously, we have:

$$
e^{g}(a+1)=(1-\lambda) e^{g}(a)
$$

\footnotetext{
${ }^{14}$ To assess the model's implications for other labor market statistics, such as the job-finding rate or the unemployment rate, one must also know the equilibrium value of $\theta$. $\pi^{0}$.

${ }^{15}$ This is of course distinct from the unconditional probability that a match is good, which we denoted by

${ }^{16}$ Note that $e^{g}(a)$ and $e^{b}(a)$ are not the measures of matches that are known by the worker and firm to be either good or bad, but rather the measure of matches that are actually good or bad (regardless of whether their type is known yet).
} 
In contrast, low quality matches remain intact only if the match does not dissolve exogenously and the quality is not revealed:

$$
e^{b}(a+1)=(1-\lambda)(1-\alpha) e^{b}(a)
$$

Define $e(a)=e^{g}(a)+e^{b}(a)$. The essence of the model is that within a given cohort, each type dissolves at constant but different rates, implying that the composition of ongoing matches varies by tenure (i.e., the share of good matches rises with tenure).

Given $e(a)$, which depends only on $\lambda, \alpha$, and $\pi^{g}$, we can easily calculate the five key statistics that are of interest for our analysis: the one quarter hazard rate, the two quarter hazard rate, the three-plus quarter hazard rate, the single quarter spell incidence rate, and the hires rate. Let $M^{*}$ be the mass of new matches that are created every period in the steady state equilibrium. As we will see below, $M^{*}$ is purely a scale factor and will disappear from all of the statistics that we compute. Steady state employment in the model, denoted by $E^{*}$, is equal to the sum of matches across the age distribution, and hence can be written as $E^{*}=\hat{e} M^{*}$ where $\hat{e}$ satisfies:

$$
\hat{e}=\sum_{a=1}^{\infty} e(a)
$$

The total number of employment spells observed in a quarter, denoted by $N^{*}$, is equal to the point-in-time employment, $\hat{e} M^{*}$, plus all of the new spells that will be created over the additional twelve weeks of the quarter:

$$
N^{*}=(\hat{e}+12) M^{*}
$$

The number of new matches initiated within a quarter is simply equal to $13 M^{*}$, since each week there is a mass $M^{*}$ of new matches created. It follows that the steady state hires rate, denoted by $h^{*}$, expressed as a fraction of total employment spells in the quarter, is given by:

$$
h^{*}=\frac{13 M^{*}}{N^{*}}=\frac{13}{(\hat{e}+12)}
$$

Next we compute the mass of one quarter spells that will be observed in steady state. In order to be a one quarter spell, the spell must start within the given quarter and not last to the next quarter. There will be 13 types of matches that meet these criteria: matches that start in the first week of the quarter and last at most 13 weeks, matches that start in the second week of the quarter and last at most twelve weeks, matches that start in the third week of the quarter and last at most 11 weeks, etc. The fraction of matches that last more than 13 weeks is $e(14)$, so the fraction of matches that start in the first week but do not survive into the next quarter is simply $1-e(14)$. Similarly, the fraction of matches that 
start in week 2 but do not last into the next quarter is $1-e(13)$. Continuing in this fashion, the total number of one quarter spells in steady state, denoted by $Q_{1}^{*}$, will be given by

$$
Q_{1}^{*}=\sum_{a=2}^{14}[1-e(a)] M^{*}
$$

and the one quarter incidence rate, denoted by $q_{1}^{*}$, is therefore:

$$
q_{1}^{*}=\frac{Q_{1}^{*}}{N^{*}}=\frac{\sum_{a=2}^{14}[1-e(a)]}{\hat{e}+12}
$$

The first quarter hazard rate is the fraction of new spells within the quarter that do not last until the next quarter. Since the number of new spells within the quarter is just equal to $13 M^{*}$, the one quarter hazard rate, denoted by $\delta_{1}^{*}$, is simply given by:

$$
\delta_{1}^{*}=\frac{Q_{1}^{*}}{13 M^{*}}=\frac{\sum_{a=2}^{14}[1-e(a)]}{13}=1-\frac{\sum_{a=2}^{14} e(a)}{13}
$$

The total mass of two quarter employment spells is given by spells that were created in the previous quarter and do not survive into the next quarter. The total number of spells that began in the previous quarter and still exist in the first period of the current quarter is equal to $\sum_{a=2}^{14} e(a) M^{*}$. In order to be counted as two quarter spells, these spells must end in the current quarter. The total number of these spells that will still exist at the start of the next quarter is $\sum_{a=15}^{27} e(a) M^{*}$. The mass of two quarter spells is thus:

$$
Q_{2}^{*}=\sum_{a=2}^{14} e(a) M^{*}-\sum_{a=15}^{27} e(a) M^{*}
$$

The hazard rate for two quarter spells is therefore given by:

$$
\delta_{2}^{*}=\frac{Q_{2}^{*}}{\sum_{a=2}^{14} e(a) M^{*}}=\frac{\sum_{a=2}^{14} e(a)-\sum_{a=15}^{27} e(a)}{\sum_{a=2}^{14} e(a)}=1-\frac{\sum_{a=15}^{27} e(a)}{\sum_{a=2}^{14} e(a)}
$$

It remains to compute the hazard rate for matches that last three-plus quarters, for which we can essentially use the same logic just used for the two quarter hazard rate, yielding:

$$
\delta_{3}^{*}=1-\frac{\sum_{a=28}^{\infty} e(a)}{\sum_{a=15}^{\infty} e(a)} .
$$

Because $e(a)$ is the sum of $e^{b}(a)$ and $e^{g}(a)$, each of which exhibits geometric decay, it follows that we have closed form expressions for all of these statistics of interest. Moreover, they depend only on the three parameters that affect $e^{b}(a)$ and $e^{g}(a)$-specifically, $\pi^{g}, \lambda$, 
Table 2: Fit of the three parameter model to the 1999 data

\begin{tabular}{lccccc}
\hline \hline & $\delta_{1}$ & $\delta_{2}$ & $\delta_{3}$ & $q_{1}$ & $h$ \\
\hline Data 1999 & 0.390 & 0.383 & 0.116 & 0.080 & 0.202 \\
$\pi^{g}=0.44, \alpha=0.158, \lambda=0.009$ & 0.390 & 0.367 & 0.116 & 0.079 & 0.202 \\
\hline
\end{tabular}

and $\alpha$.

\section{Diagnosing the Causes of Decreased Dynamism}

In this section, we use the reduced from representation of our model to shed light on what types of changes (to the three parameters) can account for the changing patterns of dynamics that we documented earlier in the paper. A key perspective of our analysis is that the tenure structure of hazard rates is an important piece of information regarding the driving forces behind the overall decrease in dynamism. For this reason our analysis will focus on the three hazard rates that we can compute from the QWI: the one quarter hazard rate, the two quarter hazard rate, and the three-plus quarter hazard rate.

In this section we ask two specific questions. First, what configuration of the three parameters $\lambda, \alpha$, and $\pi^{g}$ can account for the profile of hazard rates by tenure that existed in the QWI data at the end of the 1990s? Second, what changes in the configuration of these parameters is required in order to account for the subsequent observed changes in hazard rates by tenure. Of particular interest is to assess the extent to which changes in $\lambda$, which in our model capture changes in dynamism due to changes in separations that are uniform across the tenure distribution (and which may reflect either job destruction or worker quits) are sufficient to account for the changes observed in the data.

We begin with the first question, looking for values of $\lambda, \alpha$, and $\pi^{g}$ such that the steady state of our model can match the late 1990s values for the three hazard rates by tenure. ${ }^{17}$ Table 2 shows the results from setting $\pi^{g}=0.44, \alpha=0.158$, and $\lambda=0.009$. In addition to the three targeted moments, the table also reports two other values from the data and the model: the share of one quarter spells $\left(q_{1}\right)$ and the overall hires rate $(h)$. The table shows that our stylized model is quite successful in being able to account for the statistics observed in the data. Although we are using three parameters to fit three moments we note that the model imposes a lot of structure on the relationship between the various moments

\footnotetext{
${ }^{17}$ Our analysis will focus on the steady state properties of our model, which means that we will implicitly think of the late 1990s and mid-2010s as two different steady state outcomes of our model. In reality it could be that model parameters are changing slowly throughout the entire time period, so that the economy is not literally in steady state at any point. But as noted by Shimer (2005), the allocations during the transition closely approximate the steady state allocations.
} 
Table 3: Changes between 1999 and 2015 in the data

\begin{tabular}{lccccc}
\hline \hline & $\delta_{1}$ & $\delta_{2}$ & $\delta_{3}$ & $q_{1}$ & $h$ \\
\hline Data 1999 & 0.390 & 0.383 & 0.116 & 0.080 & 0.202 \\
Data 2015 & 0.316 & 0.335 & 0.091 & 0.049 & 0.156 \\
DData, 1999-2015 & -0.074 & -0.048 & -0.025 & -0.031 & -0.046 \\
\hline
\end{tabular}

Table 4: Accounting for the changes in the data with individual parameter changes

\begin{tabular}{lccccc}
\hline \hline & $\Delta \delta_{1}$ & $\Delta \delta_{2}$ & $\Delta \delta_{3}$ & $\Delta q_{1}$ & $\Delta h$ \\
\hline$\Delta$ Data, 1999-2015 & -0.074 & -0.048 & -0.025 & -0.031 & -0.046 \\
$\pi^{g}=0.44, \alpha=0.016, \lambda=0.009$ & -0.275 & -0.167 & 0.040 & -0.061 & -0.046 \\
$\pi^{g}=0.62, \alpha=0.158, \lambda=0.009$ & -0.106 & -0.108 & -0.003 & -0.035 & -0.046 \\
$\pi^{g}=0.44, \alpha=0.158, \lambda=0.00647$ & -0.009 & -0.023 & -0.031 & -0.02 & -0.046 \\
\hline
\end{tabular}

and cannot generate arbitrary patterns in the three hazard rates.

We now consider the second question: what configuration of changes in the three parameters would be required to account for the changes that we have observed in the data in terms of these five statistics. We begin by reporting the observed changes in the data, as shown in Table 3 .

As a first step it is useful to explore the individual impact that each of our three parameters has on these statistics. As a way to normalize the changes across parameters, and given that the overall change in dynamism is a key issue in the literature, for each parameter we calibrate the change so as to match the entire reduction in the hires rate observed in the data, and then examine the implied changes along the other dimensions. Results from this exercise are presented in Table 4.

We begin by summarizing the main effects of each change and providing some intuition for the qualitative patterns. The first exercise is a decrease in $\alpha$. Learning in our model is associated with separations, since all matches discovered to be bad will be dissolved. A decrease in $\alpha$ slows down the pace of learning, thus delaying separations. Consistent with this, we see in the second row of Table 4 that the hazard rate decreases for both one and two quarter spells but increases for the three-plus quarter spells. Intuitively, the drop in the first quarter hazard rate is larger than the drop in the second quarter hazard rate, since learning is relatively more important at low tenure when the proportion of bad matches is at its highest. Interestingly, this change results in the two quarter hazard rate being higher than the one quarter hazard rate. ${ }^{18}$ Consistent with the drop in the one quarter hazard

\footnotetext{
${ }^{18}$ While perhaps counterintuitive, this can occur because of the time aggregation implicit in the calcula-
} 
rate, the fraction of one quarter spells also decreases. This effect is partly offset by the higher hazard rates for the three-plus quarter category, since this serves to decrease the number of spells that are more than one quarter.

Next we consider an increase in $\pi^{g}$, implying a higher proportion of newly created matches are good. Intuitively, if there are relatively fewer bad matches this will decrease the overall rate of separations, and this effect should be largest at low tenures where the fraction of bad matches is highest. Consistent with this, the third row of Table 4 shows decreases in the hazard rate at all three tenure categories, with the decrease being smallest for the three-plus quarter spells. The decrease for one and two quarter spells is very similar. Given that hazard rates are lower at all tenure categories, the tenure distribution is shifted toward higher tenure levels and a smaller fraction of employment is one quarter spells. Consistent with this, we see that $q_{1}$ also decreases.

Lastly, we consider a decrease in the exogenous separation rate, $\lambda$. Although $\lambda$ is constant across the tenure distribution, the change in $\lambda$ affects the composition of matches by tenure. This is because the rate of decay for $e^{g}(a)$, i.e., $\lambda$, decreases by more than the rate of decay for $e^{b}(a)$, i.e., $\lambda+\alpha(1-\lambda)$. This composition effect varies with tenure because it accumulates over time. Nonetheless, although the change in hazard rates is smallest for one quarter spells and largest for three-plus quarter spells, the net effect is a relatively uniform change in hazard rates by tenure, especially relative to the other two parameter changes. It might seem surprising that the fraction of one quarter spells decreases so much given the very small drop in the one quarter hazard rate. This is explained by the fact that spells are lasting longer, so that there is much more mass beyond the one quarter duration. That is, as we noted when presenting the evidence in Section 2, a one quarter spell requires both a new spell to be initiated and for the spell to not survive the current quarter. The decrease in $\lambda$ serves to decrease the fraction of one quarter spells almost entirely by decreasing the incidence of new spells (relative to the rest of the distribution), rather than by affecting the hazard rate of those new spells.

The key takeaway from these exercises for our purposes is that changes in only $\alpha$ or only $\lambda$ are associated with implications that are qualitatively at odds with the observed changes in the data. In particular, in the case of a lower value of $\alpha$, the hazard rate actually increases for high tenure matches, in contrast to the decrease observed in the data. For a decrease in $\lambda$, we see that the implication is a relatively uniform drop in hazard rates by tenure, with the largest effect for three-plus quarter spells, which is also in contrast to what we observed in the data. On the other hand, the increase in $\pi^{g}$ generates changes that are

tions. The one quarter hazard rate includes spells that are created near the end of the quarter and so are only observed for a fraction of a quarter. They will appear to have a low hazard rate relative to spells created at the beginning of the quarter. All two quarter spells are necessarily in existence at the beginning of their second quarter and so the hazard for spells in their second quarter does not reflect a similar censoring. 
Table 5: Accounting for the changes in the data with changes in all three parameters

\begin{tabular}{lccccc}
\hline \hline & $\Delta \delta_{1}$ & $\Delta \delta_{2}$ & $\Delta \delta_{3}$ & $\Delta q_{1}$ & $\Delta h$ \\
\hline Data & -0.074 & -0.048 & -0.025 & -0.031 & -0.046 \\
$\pi^{g}=0.50, \alpha=0.158, \lambda=0.00735$ & -0.042 & -0.055 & -0.021 & -0.025 & -0.046 \\
$\pi^{g}=0.50, \alpha=0.140, \lambda=0.00735$ & -0.061 & -0.048 & -0.020 & -0.028 & -0.046 \\
\hline
\end{tabular}

Table 6: Decomposing the contributions of the parameter changes

\begin{tabular}{lccccc}
\hline \hline & $\Delta \delta_{1}$ & $\Delta \delta_{2}$ & $\Delta \delta_{3}$ & $\Delta q_{1}$ & $\Delta h$ \\
\hline$\pi^{g}=0.44, \alpha=0.158, \lambda=0.00735$ & -0.006 & -0.015 & -0.020 & -0.013 & -0.030 \\
$\pi^{g}=0.50, \alpha=0.14, \lambda=0.009$ & -0.054 & -0.032 & 0.001 & -0.017 & -.019 \\
\hline
\end{tabular}

qualitatively in line with what we observed in the data.

However, although the increase in $\pi^{g}$ seems to capture the key qualitative patterns in the data, it still misses the data in terms of quantitative changes. In particular, the decrease in the one and two quarters hazard rates are too large and the drop in the three-plus quarters hazard rate is too small. This suggests that a combination of an increase in $\pi^{g}$ and a decrease in $\lambda$ might better account for the observed changes. The second row of Table 5 shows the results from such a case, with $\pi^{g}$ increasing to 0.50 and $\lambda$ decreasing to 0.00735 .

This specification comes much closer to capturing the changes in hazard rates by tenure while also accounting for the overall decline in the hire rate. Looking back at the effects in Table 4, one might also conjecture that allowing for some change in $\alpha$ might improve the fit a bit further, and the final row of Table 5 shows that this is indeed the case. Lowering $\alpha$ from 0.158 to 0.140 serves to rotate the hazard rate profile, amplifying the decrease in the hazard rate of one quarter spells while modestly dampening the decrease in the other two rates.

Having uncovered a change in the three parameters $\pi^{g}, \alpha$ and $\lambda$ that can account for the changes observed in the data, our final exercise is to decompose the overall effects to illustrate the contribution of changes in $\lambda$ relative to changes in $\pi^{g}$ and $\alpha$. Table 6 shows this decomposition. The key message from this table is that the effect of changes in $\alpha$ and $\pi^{g}$ on the hires rate are roughly two thirds as large as the effect of $\lambda$. That is, while it is true that the change in $\lambda$ is the single biggest driving force behind the change in dynamics as measured by the hires rate, roughly $40 \%$ of the overall change is accounted for by changes in the parameters that are related to the learning process, $\alpha$ and $\pi^{g}$. Moreover, the changes in $\alpha$ and $\pi^{g}$ play a particularly important role in accounting for the changes in the hazard rates. 


\section{Quantitative Results}

In the previous section we argued that changes in the equilibrium value of $\pi^{g}$ are an important source of the observed changes in turnover, particularly among low-tenure employment spells. In this section we return to our model to examine what changes in the model's primitives seem best able to account for this change in $\pi^{g}$ without introducing additional counterfactual predictions.

The first step is to calibrate our model so as to be consistent with observations for the late 1990s. We will then explore the extent to which various parameter value changes can account for the observed changes that occurred between 1999 and 2015. Table 7 displays the baseline parameter values used to simulate the model. As noted previously, we assume that one model period corresponds to a week. ${ }^{19}$ The parameters near the top of the table are set to values that are common in the literature. The value of $\beta$ implies a $4 \%$ annual discount rate. We assume a Cobb-Douglas meeting function: $m(u, v)=A u^{\gamma} v^{1-\gamma}$, and set the elasticity of the meeting function with respect to unemployment, $\gamma$, equal to the workers' Nash bargaining share, $\eta$, with a value of 0.5 .

The productivity of bad and good matches are set at 0.4 and 1.0 respectively. The productivity of good matches, $y_{g}$, is a normalization. Given a value for $y_{g}$, the value of $y_{b}$ will influence the extent of wage gains with tenure that result from learning about match quality. Our choice of $y_{b}=0.4$ results in average wage growth of $22 \%$ over the first 5 years. ${ }^{20}$ This is roughly in line with the approach that we took in Pries and Rogerson (2005), where we conservatively targeted a quarter of the overall wage growth (including from sources other than learning, such as human capital accumulation) documented in Topel and Ward (1992). Given the two productivity parameters, we set the disutility of working, $b$ equal to $y_{b}$, which guarantees that matches known to be bad will be dissolved. ${ }^{21}$

Given the above values there are seven additional parameters that need to be calibrated$\alpha, \lambda, A, k_{r}, k_{h}, \pi_{0}$, and $\sigma_{\epsilon}$. We set $\alpha=0.158$ and $\lambda=0.009$, in line with the values needed to match the evidence on hazard rates and the hires rate, as detailed in section 5 . The parameters $k_{r}$ and $A$ are most closely related to the meeting rates for workers and firms. We set the parameter values to target a monthly job-finding rate of $0.474 \%$, which is the average value using data in Shimer (2012) for the six-year period surrounding our target year of 1999, and a value for the average duration of a vacancy, $1 /\left[p_{f}\left(1-H\left(\pi^{n}\right)\right)\right]$, equal to

\footnotetext{
${ }^{19}$ The short time period allows the model simulation to capture the high level of turnover that exists within a quarter (the measurement time period in the QWI).

${ }^{20}$ We calculate wage growth between the average wages in week two and at the end of year five. We use week two, rather than week one, as the starting point because wages in the first period are temporarily abnormally low due to the portion of the start-up costs that are borne by workers in the form of a reduced wage.

${ }^{21}$ While this is also the value used in Shimer (2005), we note that the interpretation is somewhat different in our model, since the average productivity of matches in our model is not exactly equal to $y_{g}$.
} 
Table 7: Baseline parameter values

\begin{tabular}{lcc}
\hline \hline Parameter & Description & Value \\
\hline$\beta$ & Weekly discount factor & $0.96^{1 / 52}$ \\
$\gamma$ & Elasticity of matching function w.r.t. unemployment & 0.5 \\
$\eta$ & Workers' Nash bargaining share & 0.5 \\
$y_{g}$ & Productivity of type $g$ jobs & 1.0 \\
$y_{b}$ & Productivity of type $b$ jobs & 0.4 \\
$b$ & Workers' disutility of work & 0.4 \\
$\lambda$ & Exogenous weekly separation rate & 0.009 \\
$\alpha$ & Weekly rate of post-match learning & 0.158 \\
$k_{r}$ & Firms' flow recruitment cost & 0.81 \\
$k_{h}$ & Start-up costs & 5.72 \\
$A$ & Efficiency of matching function & 0.279 \\
$\pi_{0}$ & Probability that match is type $g$ & 0.40 \\
$\sigma_{\epsilon}$ & Noisiness of initial signal & 1.07 \\
\hline
\end{tabular}

20 days, which is the number reported in Davis, Faberman and Haltiwanger (2013).

Direct evidence on the appropriate value for the start-up or training cost, $k_{h}$, is scarce. Silva and Toledo (2009) calibrate a DMP model that includes training and separation costs, and based on the survey evidence in Barron, Berger and Black (1997) specify a quarterly training cost equal to $11 \%$ of the output of a fully trained worker, with an average training period of 4 quarters. $^{22}$ In our model, the start-up cost is incurred entirely in the first quarter. Accordingly, to be consistent with the assumption in Silva and Toledo (2009), namely that training costs amount to $11 \%$ of a year's output by a fully productive worker, we set $k_{h}=0.11 \cdot 52 \cdot y^{g}$. One issue with this is that some matches in our model will dissolve before a significant amount of training can actually occur, so this would be an upper bound on the magnitude of the expected training costs. In view of this issue, in the appendix we conduct sensitivity analysis to examine how robust our results are to this baseline value by considering a value that is $50 \%$ lower, and we find that there is little difference in the quantitative nature of our results.

The remaining two parameters $\pi_{0}$ and $\sigma_{\epsilon}$ are closely linked to the probability that new matches end up being good, which we denoted by $\pi^{g}$ in the preceding section. $\pi_{0}$ is the unconditional probability that a meeting between a worker and firm results in a good match, so higher values of $\pi_{0}$ will necessarily increase $\pi^{g}$, which is the probability of a good match

\footnotetext{
${ }^{22}$ Silva and Toledo (2009) actually assume the training cost is equal to 0.31 of the output of a fully trained worker, but 0.20 of that reduction is due to lower actual productivity by workers who are training, as opposed to actual training costs. Because our model does not have lower productivity by workers during some training phase, we exclude that component of the cost and include only the 0.11 that is associated with actual training costs (mostly in the form of time spent training rather than producing).
} 
Table 8: Statistics of interest: data and calibrated model

\begin{tabular}{lccccccc}
\hline \hline & $\delta_{1}$ & $\delta_{2}$ & $\delta_{3}$ & $q_{1}$ & $h$ & mjfr & $u$ \\
\hline 1999 data & 0.390 & 0.383 & 0.116 & 0.080 & 0.202 & 0.474 & 0.0463 \\
Model & 0.390 & 0.367 & 0.116 & 0.079 & 0.202 & 0.474 & 0.114 \\
\hline
\end{tabular}

conditional on actually forming the match. Likewise, a higher value of $\sigma_{\epsilon}$ means the initial signal has less information, so that more matches are likely to be accepted (in the limit, as $\sigma_{\epsilon}$ gets large and the signal becomes completely uninformative, all matches are accepted and $\pi^{g} \rightarrow \pi_{0}$ ). We choose values of $\pi_{0}$ and $\sigma_{\epsilon}$ to target a value for $\pi^{g}$ of 0.44 , in line with the results of the previous section. There are many combinations of $\pi_{0}$ and $\sigma_{\epsilon}$ that can achieve this target. From the perspective of the statistics that we aimed to explain in the previous section, all that matters is the value of $\pi^{g}$, and not the values of $\pi_{0}$ and $\sigma_{\epsilon}$. However, it is possible that the reaction of the model to changes in other parameters, which we will explore below, will depend on the individual values of $\pi_{0}$ and $\sigma_{\epsilon}$ and not just on their implications for $\pi^{g}$. For our benchmark calibration we set $\pi_{0}=0.4$ and $\sigma_{\epsilon}=1.07$ and then, in the appendix, we show that our main results are robust to an alternative specification for $\pi_{0}$ and $\sigma_{\epsilon}$.

Table 8 presents the 1999 empirical values for the various statistics of interest, as well as the values from our calibrated model. In addition to the variables that we focused on in section 5, we have also included two other statistics that will be of interest: the monthly job-finding rate $(m j f r)$ and the unemployment rate. Note that the model implied values for $\delta_{1}, \delta_{2}, \delta_{3}, q_{1}$, and $h$ are identical to those in the second row of Table 2 in the preceding section, since we set/targeted the same values for $\alpha, \lambda$, and $\pi^{g}$. While the monthly jobfinding rate was a targeted statistic, the unemployment rate was not. The model implied value is substantially higher than its empirical counterpart (for the 1999 and 2015 values of the unemployment rate, we use the averages for the six-year periods surrounding our beginning and end dates, so as to reduce sensitivity to business cycle conditions in any one year). This is not too surprising: in the model, all workers who separate from their jobs must re-enter unemployment to find a new job, whereas in reality a non-trivial number of workers experience a job-to-job transition with no intervening unemployment spell. In what follows we will focus on the implications for the change in the level of unemployment. Alternatively, we could have assumed that some fraction of the exogenous separators receive new job offers immediately in order to generate a lower unemployment rate, but holding this feature constant, the implications for the change in unemployment will be very similar.

Table 9 focuses on changes that occurred between 1999 and 2015. The first row shows 
Table 9: Understanding the changes that occurred between 1999 and 2015

\begin{tabular}{|c|c|c|c|c|c|c|c|c|}
\hline & $\overline{\delta_{1}}$ & $\overline{\delta_{2}}$ & $\overline{\delta_{3}}$ & $q_{1}$ & 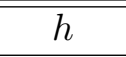 & $\overline{\pi^{g}}$ & $\overline{c m j f r}$ & $u$ \\
\hline $1999-2015 \Delta$ & -0.074 & -0.048 & -0.025 & -0.031 & -0.046 & - & -0.164 & 0.010 \\
\hline$\lambda=0.00647$ & -0.009 & -0.022 & -0.031 & -0.019 & -0.046 & -0.001 & 0.032 & -0.034 \\
\hline$\alpha=0.02$ & -0.267 & -0.156 & 0.037 & -0.060 & -0.046 & 0.041 & -0.130 & 0.009 \\
\hline$\sigma_{\epsilon}=0.44$ & -0.107 & -0.110 & -0.003 & -0.035 & -0.046 & 0.183 & -0.127 & 0.008 \\
\hline$k_{h}=33.5$ & -0.107 & -0.110 & -0.003 & -0.035 & -0.046 & 0.183 & -0.461 & 0.694 \\
\hline$A=9$ & -0.107 & -0.110 & -0.003 & -0.035 & -0.046 & 0.183 & 0.526 & -0.099 \\
\hline $\begin{array}{c}\alpha=0.14, \sigma_{\epsilon}=0.71 \\
\lambda=0.00735\end{array}$ &,-0.061 & -0.048 & -0.020 & -0.028 & -0.046 & 0.060 & -0.048 & -0.017 \\
\hline
\end{tabular}

the changes that occurred in the data. ${ }^{23}$ The changes in the hazard rates and the one quarter incidence rate $q_{1}$ are of course the same as what we previously analyzed in the reduced from analysis in 5 . We also include here the variable $\pi^{g}$, even though have no data on it, because its value is of central importance to the model's predictions for the statistics of interest. Note also that while the decline in the job-finding rate and the decline in hazard rates pull the unemployment rate in opposite directions, on net the unemployment rate increased slightly over the period.

The next rows in Table 9 show how the model's predictions for the steady state values of these statistics change as we change various model parameters. Our objective with these exercises is to see what changes in the model's parameters might be able to account for the changes observed in the data. As a way of normalizing the changes that we consider, for each parameter change we choose new values such that the new steady state equilibrium exhibits a decline in the hires rate, $h$, that is on par with what is observed in the data (i.e., a decline of 0.046). This is the same normalization that we used earlier in section 5 and seems like a natural normalization, given that the hires rate is one way of measuring the labor market turnover whose decline we seek to understand.

Based on our previous analysis, we know that achieving a good fit with the data will require a substantial increase in $\pi^{g}$. In our reduced form analysis we treated each of $\pi^{g}, \alpha$, and $\lambda$ as exogenous. But in our model, the value of $\pi^{g}$ is endogenous, and in particular will potentially respond to changes in either $\lambda$ or $\alpha$. With this in mind, we first ask whether changes in $\lambda$ or $\alpha$ alone would generate sufficient variation in $\pi^{g}$ to account for the changes found in the data.

The second and third rows of Table 9 answer this question in the negative. The decrease

\footnotetext{
${ }^{23}$ While the data in our source for the 1999 job-finding rate, i.e. Shimer (2012), ends in 2005, Engbom (2018) uses the same method as Shimer (2012) to update the data through 2015, and gets a monthly job-finding rate in 2015 of 0.31, implying a reduction of 0.164 since 1999.
} 
in $\lambda$ has effectively no impact on the value of $\pi^{g}$, so that this exercise produces results that are virtually identical to those from the reduced form analysis. Not surprisingly, the two new statistics that are added to this table show that a decrease in $\lambda$ does lead to a substantial increase in the job-finding rate and a substantial decrease in the unemployment rate. These predictions are exactly what a simple DMP model predicts. The main message is that while this change has a large impact on the incentive for firms to post vacancies, it has virtually no impact on the threshold for forming a match conditional on a meeting. To summarize, a decline in $\lambda$, by itself, cannot explain the changes observed in the data. In particular, it fails to account for the decline in hazard rates among low-tenure matches.

When we consider a decline in the rate of learning, $\alpha$, we see that there is indeed a positive impact on $\pi^{g}$, indicating that the endogenous response moves in the right direction to account for the data. Intuitively, the slower rate of learning makes it more costly to form a match with low probability of success, and as a result the match formation threshold increases. However, the size of the change in $\pi^{g}$ is quite small relative to what we found was needed in the reduced form analysis, with the result being that a change in $\alpha$ alone cannot explain the patterns in the data. We also note that this change does predict a sizeable decrease in the job-finding rate, and a modest increase in the unemployment rate. The slower learning decreases the incentive for firms to invest in vacancy posting, thereby pushing down the job-finding rate. The effect on unemployment is partially offset by the lower overall separation rate caused by the slower rate of learning.

Next we consider changes in three primitives which intuitively will have a direct impact on $\pi^{g}: \sigma_{\epsilon}, k_{h}$, and $A .{ }^{24}$ If $\sigma_{\epsilon}$ decreases then the initial signal is more informative and there will be fewer "mistakes" in terms of forming matches that are bad ( $\pi^{g}$ will increase). If $k_{h}$ increases then a bad match becomes more costly given that all matches must pay the cost $k_{h}$. If bad matches are more costly then the threshold for forming a match becomes higher (again, $\pi^{g}$ will increase). Finally, if $A$ increases, then it is easier for firms and workers to meet, allowing them to be more discerning about which matches to form.

The next three rows in Table 9 display the implications for each of these changes, once again imposing in each case that the change is sufficiently large to generate the drop in the hires rate that is observed in the data. Recall that $\delta_{1}, \delta_{2}, \delta_{3}, q_{1}$, and $h$ all depend only on $\lambda, \alpha$ and $\pi^{g}$. Because we are holding $\lambda$ and $\alpha$ constant in these exercises, we are essentially finding values for $\sigma_{\epsilon}, k_{h}$, and $A$ that achieve a new value of $\pi^{g}$ that will match the observed decline in $h$. Given that value of $\pi^{g}$, the model also generates identical changes in $\delta_{1}, \delta_{2}$, $\delta_{3}$, and $q_{1}$. But importantly, the three changes have very distinct implications for the jobfinding rate and the unemployment rate. The increase in $A$ leads to a large increase in the

\footnotetext{
${ }^{24}$ One could also consider a decrease in $k_{r}$. Not surprisingly, this turns out to be quite similar to increases in $A$ and so we do not include it in the results shown.
} 
job-finding rate and a large decrease in the unemployment rate, whereas an increase in $k_{h}$ leads to a massive decrease in the job-finding rate and an equally massive increase in the unemployment rate. Although each of these two changes has the desired effect on $\pi^{g}$, the size of the associated effects on the job-finding rate and the unemployment rate strongly suggest that they cannot be quantitatively important sources of the change in $\pi^{g} .^{25}$

In contrast, a decrease in $\sigma_{\epsilon}$ generates a decrease in the job-finding rate that is roughly in line with the magnitude of the decline found in the data, as well as a slight increase in the unemployment rate that accords with the data. Importantly, the significant decline in the job-finding rate does not translate into a large increase in the unemployment rate because the higher signal quality leads to fewer separations, thus creating an opposing effect on the unemployment rate. We conclude that a decrease in $\sigma_{\epsilon}$ seems the most promising source of the decrease in $\pi^{g}$.

Similar to what we did in the reduced form analysis, we can also consider changes in several parameters at the same time. The last row of Table 9 considers this possibility. As $\lambda$ and $\alpha$ are both exogenous parameters, we change those to the values that best fit the data in Table 5 of Section 5, i.e. $\lambda=0.00735$ and $\alpha=0.14$. Next, because $\sigma_{\epsilon}$ seemed the most promising cause of differences in $\pi^{g}$, we lower $\sigma_{\epsilon}$ to the level that is needed to account for the observed drop in the hires rate. The results in this last row of Table 9 mimic the results in Table 5 of section 5 What is new relative to the reduced form analysis is that we can now also trace out the implications for the job-finding rate and the unemployment rate. The results in the last row show that the job-finding rate falls, though not as much as in the data, and that the unemployment rate also falls modestly. We note that considering additional changes that would account for the full drop in the job-finding rate would tend to raise the unemployment rate, offsetting the modest decline reported in the table.

Our results are also of interest in the context of a recent literature that seeks to estimate changes in matching efficiency - see, for example, Hornstein and Kudlyak (2016) or Hall and Schulhofer-Wohl (2018). Those papers conclude that there was a decline in matching efficiency over the period 2000-2015, on the order of 15-20\%. Our model provides an alternative interpretation of the same underlying facts. In particular, those papers interpret the matching function as providing a stable mapping between inputs (vacancies and unemployed workers) and outputs (new matches that are formed). In our model, there is a stable relationship between inputs into the matching function and meetings, but any parameters that change the equilibrium threshold rule for match formation will affect the mapping between inputs in the matching function and the formation of new matches. In particular,

\footnotetext{
${ }^{25}$ Put somewhat differently, although changes in each of $A$ and $k_{h}$ can lead to increases in $\pi^{g}$, the elasticity of these effects are sufficiently small that it takes very large changes in these variables to generate the changes in $\pi^{g}$ required to account for the data, and these large changes produce counterfactually large effects on other aspects of the equilibrium.
} 
our model interprets the same observations as these papers as the results of workers and firms being more selective when forming matches, as opposed to workers and firms having a harder time finding partners. In fact, for the simulation in Table 9 that considers only a decline in $\sigma_{\epsilon}$, the equilibrium meeting rate between workers and firms actually increases, but the fraction of meetings that result in new matches being formed goes down considerably. The efficiency parameter that one would measure from the relationship between the job-finding rate and the $\mathrm{v} / \mathrm{u}$ ratio would decline, but this would be attributable to better screening as opposed to lower efficiency in the matching process.

\section{Conclusion}

A growing literature has documented the declining "dynamism" of the US economy as reflected by declines in various measures of worker, job, and firm turnover and sought to understand the driving forces behind these declines. In this paper, we have argued that a key aspect of the decline in worker turnover is accounted for by a decline in short duration employment spells, and that this decline is pervasive across different types of workers and firms. This suggests a need to focus on an explanation for lower turnover that simultaneously predicts a large decrease in the share of short duration employment spells. Our analysis of a standard DMP model extended to account for the salient facts about hazard rates by employment spell tenure leads us to conclude that firms and workers have become better able to identify low productivity matches ex ante, thereby increasing the average quality of those matches that do get formed. This explanation is consistent with recent empirical evidence on human resource practices. More generally, our analysis suggests that better matching accounts for about $40 \%$ of the overall decline in the level of worker turnover observed in the US over the last two decades. This implies that there is not a singular explanation for the overall decline in worker turnover. Importantly, whereas improved matching can be understood as a positive development, other factors at work may reflect negative developments. A key message from our analysis is that explanations for the changing dynamism of the US labor market need to pay attention to the changing distribution of employment spells by tenure. 


Employment Change, Individual
\begin{tabular}{|l|c|c|c|c|c|c|c||c|c|c|c|c|}
\hline Measures & -5 & -4 & -3 & -2 & -1 & $\boldsymbol{t}$ & +1 & +2 & +3 & +4 & +5 \\
\hline Hires All & & & & & $\mathbf{X}$ & & & & & & \\
\hline Hires New & & $\mathbf{X}$ & $\mathbf{X}$ & $\mathbf{X}$ & $\mathbf{X}$ & & & & & & \\
\hline Hires Recalls & & - & - & - & $\mathbf{X}$ & & & & & & \\
\hline All Hires, End-of-Quarter employed & & & & & $\mathbf{X}$ & & & & & & \\
\hline Separations, Beginning-of-Quarter Employed & & & & & & & $\mathbf{X}$ & & & & \\
\hline Separations & & & & & & & $\mathbf{X}$ & & & & \\
\hline Hires All (Stable) & & & & $\mathbf{X}$ & & & & & & & \\
\hline Hires New (Stable) & $\mathbf{X}$ & $\mathbf{X}$ & $\mathbf{X}$ & $\mathbf{X}$ & & & & & & & \\
\hline Separations (Stable) & & & & & & & $\mathbf{X}$ & & & & \\
\hline Separations (Stable), next quarter & & & & & & & & $\mathbf{X}$ & & & \\
\hline
\end{tabular}

Figure 11: Various measures of employment changes in the QWI.

\section{A Data}

We utilize the version of the 2018Q2 release of the QWI public use data that includes only private sector employment. Our analysis focuses on 30 states, which together account for over $65 \%$ total U.S. employment: CA, CO, CT, FL, GA, HI, ID, IL, IN, KS, LA, MD, ME, MN, MO, MT, NC, ND, NJ, NM, NV, PA, RI, SC, SD, TN, TX, VA, WA, and WV. For those 30 states, data for the variables that we utilize are available from 1999Q1 through 2015Q2. To broaden to a larger set of states would require that we shorten the coverage period, as data for other states is not available until later dates.

The QWI public use data give information on individual-level employment and employment changes, aggregated across individuals by worker categories that include sex, age, race, and education, and firm categories that include industry, firm size, firm age, and state.

Figure 11 displays the different measures of individual-level employment changes that are available in the QWI public use data. From this information, we can identify the various rates and measures utilized in the paper. The overall number of hires in period $t, H_{t}$ is given by "Hires All" (QWI variable name HirA). We can make this into a rate by dividing by a measure of employment. There are two possible measures of employment: Emp, which is the average of the beginning-of-period employment and the end-of-period employment, or EmpTotal, which is the total number of employment spells in the period, $N_{t}$ (and thus includes spells that either end in the period, begin in the period, or both end and begin in the period, in addition to the spells that continue through the entire period). Although, EmpTotal always exceeds Emp and thus will result in a lower level for the rates that it is used to calculate, the trends (which is the primary focus of the paper) that result from use of the two measures of employment are very similar. Thus, using EmpTotal as the employment measure, we calculate the hires rate $h_{t}$ as:

$$
h_{t}=\frac{\operatorname{HirA}_{t}}{\text { EmpTotal }_{t}}
$$


In the paper, in section 3.2 , we contrast $h_{t}$ with a hires rate that focuses on hires of workers who had no employment with the hiring firm in the previous four quarters. For that measure, we use "Hires new" (QWI variable name HirN) in the numerator.

We can also calculate a rate of separations by dividing total separations $S_{t}$ (QWI variable name Sep) by EmpTotal:

$$
s_{t}=\frac{\operatorname{Sep}_{t}}{\text { EmpTotal }_{t}}
$$

Employment spells that last just one quarter in period $t, Q_{1 t}$, are identified as "Hires All" (HirA) minus "All hires, end-of-quarter employed" (HirAEnd). This is made into an incidence rate by dividing by EmpTotal:

$$
q_{1 t}=\frac{\operatorname{HirA}_{t}-\operatorname{HirAEnd}_{t}}{\text { EmpTotal }_{t}}
$$

Employment spells that last two periods can also be measured. The lag of HirAEnd tells us the number of workers that began employment in period $t-1$ and remained employed in period $t$, and possibly longer. The variable "Hires All (stable)" (QWI variable name HirAS) tells us the number of workers that began employment in period $t-1$ and then continued in that same job in periods $t$ and $t+1$. Thus, if we subtract HirAS from the lag of HirAEnd we obtain the number of workers who were in their second period of employment in period $t$, but did not remain employed in that same job in period $t+1$. That is, we can identify two quarter employment spells that end in period $t, Q_{2 t}$. We can similarly make this into an incidence rate by dividing by the total number of employment spells, $N_{t}$, in that period:

$$
q_{2 t}=\frac{\operatorname{HirAEnd}_{t-1}-\operatorname{HirAS}_{t}}{\text { EmpTotal }_{t}}
$$

In addition to these incidence rates, we can also calculate a hazard rates for the three tenure categories. For first-quarter employment spells the hazard rate is given by

$$
\delta_{1 t}=\frac{\operatorname{HirA}_{t}-\operatorname{HirAEnd}_{t}}{\operatorname{HirA}_{t}}
$$

and for second-quarter employment spells the hazard rate is:

$$
\delta_{2 t}=\frac{\operatorname{HirAEnd}_{t-1}-\operatorname{HirAS}_{t}}{\operatorname{HirAEnd}_{t-1}} .
$$

The hazard rate among employment spells with three-plus quarters of tenure is calculated by subtracting one quarter spells and two quarters spells from total separations, and dividing 
Table 10: Alternative specification of $\pi_{0}$ and $\sigma_{\epsilon}$

\begin{tabular}{lcccccccc}
\hline \hline & $\delta_{1}$ & $\delta_{2}$ & $\delta_{3}$ & $q_{1}$ & $h$ & $\pi^{g}$ & mjfr & $u$ \\
\hline $1999-2015 \Delta$ & -0.074 & -0.048 & -0.025 & -0.031 & -0.046 & - & -0.164 & 0.010 \\
\hline$\lambda=0.00647$ & -0.008 & -0.021 & -0.031 & -0.019 & -0.046 & -0.002 & 0.032 & -0.034 \\
$\alpha=0.021$ & -0.265 & -0.153 & 0.036 & -0.059 & -0.046 & 0.045 & -0.135 & 0.011 \\
$\sigma_{\epsilon}=0.43$ & -0.107 & -0.110 & -0.003 & -0.035 & -0.046 & 0.183 & -0.119 & 0.005 \\
$k_{h}=32.5$ & -0.107 & -0.110 & -0.003 & -0.035 & -0.046 & 0.183 & -0.452 & 0.606 \\
$A=7.1$ & -0.107 & -0.110 & -0.003 & -0.035 & -0.046 & 0.183 & 0.523 & -0.096 \\
$\alpha=0.14, \sigma_{\epsilon}=0.68,-0.060$ & -0.046 & -0.020 & -0.027 & -0.046 & 0.058 & -0.041 & -0.018 \\
$\quad$ & & & & & & & & \\
\hline
\end{tabular}

by the total number of employment spells with three-plus quarters of tenure:

$$
\delta_{3 t}=\frac{\operatorname{Sep}_{t}-\left(\operatorname{HirA}_{t}-\operatorname{HirAEnd}_{t}\right)-\left(\operatorname{HirAEnd}_{t-1}-\operatorname{HirAS}_{t}\right)}{\text { EmpTotal }_{t}-\operatorname{HirA}_{t}-\operatorname{HirAEnd}_{t-1}}
$$

We also utilize a measure of job creation - the change in firm-level employment, aggregated across all firms whose employment grew in the quarter - which is given by the QWI variable FrmJbGn. We express this as a rate by dividing by $N_{t}$, the total number of employment spells in the period:

$$
\frac{\text { Frm JbGn }_{t}}{\text { EmpTotal }_{t}}
$$

We seasonally adjust all variables in the paper by regressing on quarterly dummies and removing those quarterly components. We directly seasonally adjust the variable of interest, as opposed to seasonally adjusting each data component individually before constructing the variable.

\section{B Robustness Exercises}

We report here the results of two exercises that examine how robust our results are to alternative parameter values. The first exercise concerns the fact that many different combinations of $\pi_{0}$ and $\sigma_{\epsilon}$ could achieve the targeted value of $\pi^{g}$ in the baseline calibration. Here we consider an alternative pair of values for $\pi_{0}$ and $\sigma_{\epsilon}$ and assess whether our primary findings are affected in a significant way. The second exercise concerns the calibration of the training cost parameter $k_{h}$.

We begin with the choice of $\pi_{0}$ and $\sigma_{\epsilon}$. In our baseline calibration we set $\pi_{0}=0.40$. Here we set $\pi_{0}=0.38$, which then requires that we reduce the noise of the initial signal to $\sigma_{\epsilon}=0.95$ in order to hit the desired value of $\pi^{g}$. That is, a smaller fraction of matches are 
Table 11: Alternative specification of $k_{h}$

\begin{tabular}{lcccccccc}
\hline \hline & $\delta_{1}$ & $\delta_{2}$ & $\delta 3$ & $q_{1}$ & $h$ & $\pi^{g}$ & mjfr & $u$ \\
\hline $1999-2015 \Delta$ & -0.074 & -0.048 & -0.025 & -0.031 & -0.046 & - & -0.164 & 0.010 \\
\hline$\lambda=0.00647$ & -0.009 & -0.022 & -0.031 & -0.020 & -0.046 & -0.000 & 0.021 & -0.032 \\
$\alpha=0.02$ & -0.261 & -0.149 & 0.034 & -0.059 & -0.046 & 0.059 & -0.135 & 0.011 \\
$\sigma_{\epsilon}=0.376$ & -0.107 & -0.110 & -0.003 & -0.035 & -0.046 & 0.183 & -0.123 & 0.006 \\
$k_{h}=29.1$ & -0.107 & -0.110 & -0.003 & -0.035 & -0.046 & 0.183 & -0.417 & 0.378 \\
$A=4.48$ & -0.107 & -0.110 & -0.003 & -0.035 & -0.046 & 0.183 & 0.525 & -0.098 \\
$\alpha=0.14, \sigma_{\epsilon}=0.59,-0.060$ & -0.047 & -0.020 & -0.028 & -0.046 & 0.059 & -0.046 & -0.017 \\
\multicolumn{1}{l}{$\lambda=0.00735$} & & & & & & & & \\
\hline
\end{tabular}

of good quality, but the lower level of noise in the signal means that workers and firms can better assess match quality. This combination again implies that $\pi^{g}=0.44$. Matching the same targets as before requires that we also re-calibrate $A$ and $k_{r}$, which we change to 0.308 and 0.81 respectively, in order to achieve a monthly job-finding rate of 0.474 and an average vacancy duration of 20 months. Because we match the same targets as in the baseline calibration, the model delivers the same values for the calibrated steady state. Table 10 repeats the exercises from Table 9 . The message from this exercise is that the key results are effectively unchanged. In particular, we again conclude that changes in $k_{h}$ and $A$ result in implausible changes in the job-finding rate and unemployment rate, whereas a reduction in $\sigma_{\epsilon}$ has the most promise in accounting for an increase in $\pi^{g}$.

Next we consider an alternative value of $k_{h}$. We previously noted that assuming all of the start-up cost was incurred in the first period might overestimate the extent of expected start-up costs if they are actually incurred over several periods and matches found to be bad were terminated. With this in mind we consider a specification with a much smaller value for $k_{h}$. In the baseline we assumed $k_{h}=5.72$, and here we consider half that amount, $k_{h}=2.86$. We leave $\pi_{0}=0.4$ as in the benchmark but $\sigma_{\epsilon}$ is now set to 0.83 in order to match the target $\pi^{g}=0.44$. We once again recalibrate the values of $A$ and $k_{r}$ to 0.266 and 0.917 respectively in order to hit the targeted monthly job-finding rate of 0.47 and the average vacancy duration of 20 weeks.

Table 11 repeats the exercises from Table 9. Once again, these results support the same message as the baseline calibration. In particular, it remains the case that adjusting $k_{h}$ to achieve the desired change in $\pi^{g}$ leads to implausibly large increases in the unemployment rate and declines in the job-finding rate. We again conclude that changes in $\sigma_{\epsilon}$ are the most promising source of changes in $\pi^{g}$. 


\section{References}

Akcigit, Ufuk, and Sina Ates. 2019. "What Happened to U.S. Business Dynamism?" University of Chicago Working Paper.

American Staffing Association. 2018. "ASA Staffing Employment and Sales Survey." $\quad$ https://americanstaffing.net/staffing-research-data/asa-data-dashboard/asaemployment-sales/.

Autor, David, and David Scarborough. 2008. "Does Job Testing Harm Minority Workers? Evidence from Retail Establishments." The Quarterly Journal of Economics, 123(1): 219-277.

Barron, John M., Mark C. Berger, and Dan A. Black. 1997. On-the-Job Training. Kalamazoo, MI:Upjohn Institute for Employment Research.

Davis, Steven J., and John Haltiwanger. 2014. "Labor Market Fluidity and Economic Performance." National Bureau of Economic Research, Inc NBER Working Papers 20479.

Davis, Steven J., R. Jason Faberman, and John C. Haltiwanger. 2013. "The Establishment-Level Behavior of Vacancies and Hiring." The Quarterly Journal of Economics, 128(2): 581-622.

Decker, Ryan A., John Haltiwanger, Ron S. Jarmin, and Javier Miranda. 2017. "Declining Dynamism, Allocative Efficiency, and the Productivity Slowdown." American Economic Review, 107(5): 322-26.

Engbom, Niklas. 2018. "Firm and Worker Dynamics in an Aging Labor Market." Society for Economic Dynamics 2018 Meeting Papers 1009.

Hall, Robert E., and Sam Schulhofer-Wohl. 2018. "Measuring Job-Finding Rates and Matching Efficiency with Heterogeneous Job-Seekers." American Economic Journal: Macroeconomics, 10(1): 1-32.

Hoffman, Mitchell, Lisa B Kahn, and Danielle Li. 2017. "Discretion in Hiring*." The Quarterly Journal of Economics, 133(2): 765-800.

Hornstein, Andreas, and Marianna Kudlyak. 2016. "Estimating Matching Efficiency with Variable Search Effort.” Federal Reserve Bank of Richmond Working Paper 16-13.

Hyatt, Henry, and James Spletzer. 2013. "The recent decline in employment dynamics." IZA Journal of Labor Economics, 2(1): 1-21. 
Hyatt, Henry R., and James R. Spletzer. 2017. "The recent decline of single quarter jobs." Labour Economics, 46(C): 166-176.

Mercan, A. Yusuf. 2017. "Fewer but Better: The Decline in Job Mobility and the Information Channel." University of Melbourne Working Paper.

Molloy, Raven, Christopher L. Smith, Riccardo Trezzi, and Abigail Wozniak. 2016. "Understanding Declining Fluidity in the U.S. Labor Market." Brookings Papers on Economic Activity, 183-237.

Pries, Michael, and Richard Rogerson. 2005. "Hiring Policies, Labor Market Institutions, and Labor Market Flows." Journal of Political Economy, 113(4): 811-839.

Pugsley, Benjamin Wild, and Aysegul Sahin. 2018. "Grown-up Business Cycles." The Review of Financial Studies, 32(3): 1102-1147.

Shimer, Robert. 2005. "The Cyclical Behavior of Equilibrium Unemployment and Vacancies." American Economic Review, 95(1): 25-49.

Shimer, Robert. 2012. "Reassessing the Ins and Outs of Unemployment." Review of Economic Dynamics, 15(2): 127-148.

Silva, José Ignacio, and Manuel Toledo. 2009. "Labor Turnover Costs And The Cyclical Behavior Of Vacancies And Unemployment." Macroeconomic Dynamics, 13(S1): 7696.

Topel, Robert H., and Michael P. Ward. 1992. "Job Mobility and the Careers of Young Men." The Quarterly Journal of Economics, 107(2): 439-479. 